\title{
Commentary \\ Commentary: Iconoclastic Reflections on the 'Safety' of Polyunsaturated Fatty Acid-Rich Culinary Frying Oils: Some Cautions regarding the Laboratory Analysis and Dietary Ingestion of Lipid Oxidation Product Toxins
}

\author{
Martin Grootveld ${ }^{1, *(\mathbb{D}}$, Benita C. Percival ${ }^{1}$, Sarah Moumtaz ${ }^{1}$, Miles Gibson ${ }^{1}$, Katy Woodason ${ }^{1}$, Azeem Akhtar ${ }^{1}$, \\ Michael Wawire ${ }^{2}$, Mark Edgar ${ }^{3}$ and Kerry L. Grootveld ${ }^{1}$ \\ 1 Leicester School of Pharmacy, De Montfort University, The Gateway, Leicester LE1 9BH, UK; \\ P11279990@alumni365.dmu.ac.uk (B.C.P.); sarah.moumtaz@gmail.com (S.M.); \\ miles.gibson@dmu.ac.uk (M.G.); katy.woodason@dmu.ac.uk (K.W.); azeem.akhtar@dmu.ac.uk (A.A.); \\ k.grootveld@icloud.com (K.L.G.) \\ 2 Department of Food Science and Technology, School of Food and Nutrition Sciences (SOFNUS), \\ Jomo-Kenyatta University of Agriculture and Technology, P.O. Box 62000, Nairobi 00200, Kenya; \\ wawire@agr.jkuat.ac.ke \\ 3 Department of Chemistry, University of Loughborough, Epinal Way, Loughborough LE11 3TU, UK; \\ m.edgar@lboro.ac.uk \\ check for \\ updates \\ * Correspondence: mgrootveld@dmu.ac.uk; Tel.: +44-(0)116-250-6443
}

Citation: Grootveld, M.; Percival, B.C.; Moumtaz, S.; Gibson, M.; Woodason, K.; Akhtar, A.; Wawire, M.; Edgar, M.; Grootveld, K.L. Commentary: Iconoclastic Reflections on the 'Safety' of Polyunsaturated Fatty Acid-Rich Culinary Frying Oils: Some Cautions regarding the Laboratory Analysis and Dietary Ingestion of Lipid Oxidation Product Toxins. Appl. Sci. 2021, 11, 2351. https://doi.org/10.3390/app11052351

Academic Editor:

Alessandra Biancolillo

Received: 25 December 2020

Accepted: 1 March 2021

Published: 6 March 2021

Publisher's Note: MDPI stays neutral with regard to jurisdictional claims in published maps and institutional affiliations.

Copyright: (c) 2021 by the authors. Licensee MDPI, Basel, Switzerland. This article is an open access article distributed under the terms and conditions of the Creative Commons Attribution (CC BY) license (https:// creativecommons.org/licenses/by/ $4.0 /)$.

\begin{abstract}
Continuous or frequent ingestion of fried foods containing cytotoxic/mutagenic/genotoxic lipid oxidation products (LOPs) may present significant human health risks; such toxins are generated in thermally stressed polyunsaturated fatty acid (PUFA)-rich culinary frying oils (CFOs) during standard frying practices. Since monounsaturated and saturated fatty acids (MUFAs and SFAs, respectively) are much less susceptible to peroxidation than PUFAs, in this study CFOs of differential unsaturated fatty acid contents were exposed to laboratory-simulated shallow-frying episodes (LSSFEs). Firstly, we present a case study exploring the time-dependent generation of aldehydic LOPs in CFO products undergoing LSSFEs, which was then used to evaluate the relative potential health risks posed by them, and also to provide suitable recommendations concerning their safety when used for frying purposes. Sunflower, rapeseed, extra-virgin olive and coconut oils underwent LSSFEs at $180{ }^{\circ} \mathrm{C}$ : Samples were collected at 0-90 min time-points ( $n=6$ replicates per oil). Aldehydes therein were determined by highresolution ${ }^{1} \mathrm{H}$ NMR analysis at 400 and $600 \mathrm{MHz}$ operating frequencies. For one of the first times, CFO LOP analysis was also performed on a non-stationary $60 \mathrm{MHz}$ benchtop NMR spectrometer. ${ }^{1} \mathrm{H}$ NMR analysis confirmed the thermally promoted, time-dependent production of a wide range of aldehydic LOPs in CFOs. As expected, the highest levels of these toxins were produced in PUFA-rich sunflower oil, with lower concentrations formed in MUFA-rich canola and extra-virgin olive oils; in view of its very high SFA content, only very low levels of selected aldehyde classes were generated in coconut oil during LSSFEs. Secondly, ${ }^{1} \mathrm{H}$ NMR results acquired are discussed with regard to the suitability and validity of alternative, albeit routinely employed, spectrophotometric methods for evaluating the peroxidation status of CFOs and lipid-containing foods. Thirdly, an updated mini-review of the toxicological properties of and intake limits for LOPs, and deleterious health effects posed by their ingestion, is provided. In conclusion, exposure of PUFA-rich CFOs to high-temperature frying practices generates very high concentrations of aldehydic LOP toxins from thermally promoted, $\mathrm{O}_{2}$-powered, recycling peroxidation processes; these toxins penetrate into and hence are 'carried' by fried foods available for human consumption. Such toxins have the capacity to contribute towards the development and progression of non-communicable chronic diseases (NCDs) if cumulatively ingested by humans.
\end{abstract}

Keywords: culinary frying oils; seed oils; frying practices; lipid peroxidation; lipid oxidation products; toxic aldehydes; high-resolution NMR analysis; food toxicology; population toxicology; genotoxicity; mutagenicity and carcinogenicity; cardiovascular diseases 


\section{Introduction}

Polyunsaturated fatty acids (PUFAs) encompass both omega- 6 and omega-3 fatty acids (FAs, $\omega-6$ and $\omega-3$ FAs, respectively), which are essential, and hence dietary sources of them are critical requirements for humans [1]. To date, PUFAs have been considered as functional foods and nutraceuticals, and a wide range of research investigations have reported their beneficial health effects through their involvements in key biochemical pathways, which offer significant cardioprotective roles in view of their anti-arrhythmic, anti-atherogenic, anti-inflammatory, anti-thrombotic and hypolipidemic effects. In turn, such properties may diminish the risks of cardiovascular diseases (CVDs), osteoporosis, diabetes, and questionably cancer too, for example. Such activities appear to be promoted by the abilities of these fatty acids (FAs) to influence blood plasma lipoprotein levels, biological membrane fluidities, eicosanoid generation, membrane enzyme and receptor activities, along with their favourable blood pressure and mineral metabolising properties.

Moreover, nutritionists continue to recommend PUFA-rich vegetable oils as an important component of a healthy diet in view of their high contents of essential FAs such as linoleic and linolenic acids (FAO/WHO Expert Consultation Report, 2010 [2]). Notwithstanding, the content and distribution of such FAs is critically dependent on the plant sources of edible frying oils, together with technological processes employed for their commercial production.

Many currently available scientific literature reports provide a high level of supporting evidence for the beneficial health effects exerted by dietary PUFAs and monounsaturated fatty acids (MUFAs), whereas saturated fatty acids (SFAs) in general have a bad reputation and press $[3,4]$. However, it is certainly clear that different homologues within the same FA classes elicit differing physiological effects. Indeed, this often used very broad SFA/MUFA / $\omega-6$ PUFA/ $\omega-3$ PUFA classification system fails to consider the health properties, positive or negative, of individual homologue members within each of these classifications (linoleic acid is a very common $\omega-6$ PUFA, and linolenic and docosahexaenoic acids are common $\omega-3$ PUFAs). For example, medium-chain SFAs, present at high levels in coconut oil, offer powerful anti-inflammatory effects which are now quite widely reported [5]. Notwithstanding, absolute conclusive evidence regarding the benefits of replacing dietary SFAs with PUFAs and/or MUFAs in diminishing disease risks, notably those of CVDs, remains unavailable [6].

However, potential associations between the reported daily intakes (RDIs) of SFAs, MUFAs and PUFAs (as \% $\mathrm{E}_{\mathrm{RDI}}$ ) for adults and coronary heart disease (CHD) and cardiovascular disease (CVD) mortalities, in a total of twelve countries [2], were not confirmed by Orsavova et al., in 2015 [7]. Nonetheless, investigation [7] is of some concern since it only involved empirical Spearman's rank bivariate correlation assessments, when clearly it should be represented by a mega-dimensional multivariate (MV) experimental model involving the consideration of a potentially large number of contributing and/or conditioning variables. Moreover, chronic non-communicable diseases (NCDs) such as CVDs are, of course, both multigenic and multifactorial.

The 2015-2020 Dietary Guidelines for Americans [8] states that 'Strategies to shift intake [from solid fats to oils] include using vegetable oil in place of solid fats (butter, stick margarine, shortening, lard, coconut oil) when cooking ... ', presumably at temperatures employed for frying or other thermally-mediated cooking episodes. Globally, the recommendation of health and nutrition authorities that such culinary frying oils (CFOs) are the most 'healthfriendly' prerogatives available for food frying applications may present a challenging issue, since these now directly conflict with a plethora of scientific results acquired by many research groups globally. Indeed, for many of the valuable population health properties potentially offered by pure unmodified, uncontaminated and unoxidized dietary PUFAcontaining acylglycerols, the converse is the case for dietary LOPs, especially aldehydes and epoxy-FAs derived from the peroxidation of unsaturated fatty acids (UFAs) [9-11]; very high levels of these toxins are generated during the exposure of UFA-rich (most especially PUFA-rich) edible oils to high temperature frying practices [12,13]. 
Although many of the reports previously focused on the 'health-friendly' properties of dietary PUFAs purport to reassure us of the 'safety' of vegetable and vegetable seed oils for human consumption, many of them appear to have completely neglected how the 'health index' parameters which they have carefully selected to report (specifically, lipid hydroperoxide and associated conjugated diene concentration values within permissible limits, and also perhaps negligible levels of trans-fatty acids (TFAs)) may be substantially changed when such oils are exposed to high temperatures commonly associated with frying or cooking practices (ca. $180{ }^{\circ} \mathrm{C}$ ), which represent the prime or major purpose for which they are available for purchase by consumers. However, although some researchers focused in this area tend to unconditionally (and perhaps conveniently) disregard a large number of important considerations regarding the deleterious generation of highly cytotoxic and genotoxic LOPs in these oils, most especially when they are subjected to such frying/cooking episodes (or alternatively, when stored for prolonged periods of time at ambient temperature and/or exposed to light in the presence of atmospheric $\mathrm{O}_{2}$ ), increasing numbers of nutritionists and food scientists now take an alternative viewpoint, i.e., they no longer 'shun' this very important public health concern. Notwithstanding, systematic reviews, combined with meta-analyses of prospective cohort studies, such as that reported by Farvid et al. [3], unfortunately appear to consistently retain all possible forms of PUFA intakes, i.e., that of the naturally occurring, unheated and unperoxidised category, and that arising from foods exposed to high temperature frying practices in PUFA-rich CFOs (i.e., those contaminated by LOPs, especially in used or reused oils), within the same broad variable classification system. A similar strong argument can be made for differential types of FA homologues within the broad SFA, MUFA and PUFA classifications themselves. For example, the $\omega-3$ FA linolenic acid peroxidises more readily than the linoleic acid [14], and medium-chain SFAs, which are present at high levels in coconut oil, appear to offer a range of 'health-friendly' properties [5].

The peroxidation of UFAs during frying or cooking episodes is an extremely complex chemical degradation process which involves very highly reactive free radical species, and singlet oxygen $\left({ }^{1} \mathrm{O}_{2}\right)$. Mechanisms available for this process primarily feature the oxidative conversion of such UFAs to primary LOPs, commonly described as lipid hydroperoxides (also known as hydroperoxymonoenes and conjugated hydroperoxydienes according to their FA sources, and abbreviated HPMs and CHPDs, respectively), a process sequentially followed by their fragmentation to secondary LOPs, the latter including extremely toxic aldehydes in particular [11,15]. Further HPM and CHPD degradation products include alcohols, ketones, oxo-acids, alkanes and alkenes [11,14,16,17]. Moreover, epoxy-FAs such as 9,10-epoxy-12-octadecenoate, which is also known as leukotoxin, also arise from the oxidation of UFAs [18]. The basis of our research group's previously reported research findings is that SFA acylglycerols generate very little or no toxic LOPs when heated according to standard frying practices in the presence of atmospheric $\mathrm{O}_{2}$, whereas on a mole-for-mole equivalence scale, those containing PUFAs produce very high levels of these toxins when exposed to thermally facilitated peroxidative assaults, as expected $[12,13,19,20]$. However, HPM-generating MUFAs are much more resistant to oxidation than PUFAs, and hence they give rise to lower levels of only particular LOPs when heated in this manner, and generally only after exposure to prolonged thermal stressing episodes at standard frying temperatures. Therefore, the order of toxic LOP production in culinary oils is PUFAs $>>$ MUFAs $>>>>>$ SFAs, and hence healthwise, PUFA-rich CFOs are generally the worst possible choice for use as frying media, especially for repeated frying episodes [13]. Indeed, the relative oxidative susceptibilities of these lipid molecules are 1:100:1200:2500 for 18-carbon chain length FAs containing 0:1:2:3 carbon-carbon double bonds (i.e., $>\mathrm{C}=\mathrm{C}<$ functions), respectively [14]. Moreover, the rate of thermally-induced degradation of CHPDs or HPMs to the above series of smaller molecular fragments also increases with increasing FA unsaturation status, i.e., it is in the order linolenoyl- > linoleoyl- > > oleoylglycerols [16]. 
Our previously reported extensive high-resolution NMR analysis investigations of the oxidative deterioration of PUFA-rich CFOs during standard frying practices have demonstrated the heat-promoted generation of very high levels of highly toxic aldehydes, in addition to their HPM and CHPD precursors, in such products $[12,19,20]$, and these results have been available to the scientific community since 1994 [19]. Indeed, samples of repeatedly used CFOs collected from domestic kitchens, fast-food retail outlets and restaurants, have confirmed their generation at high concentrations during 'on-site' frying practices; these results have been repeatedly ratified in laboratory simulations of both deep- and shallow-frying processes [11]. We can also employ these NMR techniques to monitor the corresponding oxidative degradation of culinary oil PUFAs and MUFAs during such standard frying/cooking practices $[12,13,19,20]$. These studies have been repeated, replicated, and further manifested by many research group laboratories worldwide, for example investigations reported in [21,22]. Curiously, as noted above, until recently these major population public health concerns have received only limited attention from the food industry and public health researchers.

Furthermore, in 1998 we first revealed that typical chemically reactive $\alpha, \beta$-unsaturated aldehydes produced during the thermal stressing of culinary oils according to standard frying practices are absorbed from the gut into the systemic circulation in vivo [23], where they have access and may cause damage to cells, tissues and essential organs. Further investigations performed in both animal model systems and humans have demonstrated an enhanced urinary excretion of these toxins following their ingestion [24,25].

Aldehydic LOPs act as potent toxins since they are extremely chemically reactive. Indeed, they cause damage to critically important biomolecules such as DNA: since they are powerful electrophilic alkylating agents, $\alpha, \beta$-unsaturated aldehydes readily alkylate DNA base adducts, and this serves to explain their mutagenic, genotoxic and carcinogenic properties. Moreover, these secondary LOPs have the ability to form covalent adducts with many proteins via Schiff base or Michael addition reactions [12,23], and these induce significant structural and conformational changes in these biomacromolecules which, in turn, serve to impair their biological functions.

In view of these considerations, such secondary LOPs give rise to a broad spectrum of concentration-dependent cellular stresses. The deleterious toxicological properties and health effects of these aldehydes represent one major consequential focus of this communication, and these include their adverse influence on critical metabolic pathways (for example, [26]); promotion and perpetuation of atherosclerosis and cardiovascular diseases [23,27-29]); mutagenic and carcinogenic properties [30-35]; teratogenic actions (embryo malformations during pregnancy [36]; exertion of striking pro-inflammatory effects [10,37]; induction of gastropathic properties (peptic ulcers) following dietary ingestion [38]; neurotoxic actions, including those of 4-hydroxy-trans-2-nonenal (HNE) and -hexenal (HHE) [39]; and the adverse stimulation of significant increases in systolic blood pressure [40]. Further deleterious health effects include chromosomal aberrations, reflecting their clastogenic potential, and sister chromatid exchanges and point mutations, in addition to cell damage and death [41,42]. Moreover, HNE also acts as a toxic second messenger [43]. Therefore, the 'safety' of vegetable-based culinary oils, seed or otherwise [44], remains highly questionable, particularly when they have been thermally stressed at high frying temperatures.

In many developing countries such as Kenya, Tanzania and Uganda, the toxicities of used frying oils could be exacerbated by the fact that the frying of foods by commercial enterprises is highly unregulated, and a high proportion of such frying activities are performed by vendors at the roadside, where the oils are also exposed to environmental factors, such as direct sunlight, heat, dust and moisture during storage, preparation and frying episodes. This indicates that even before the commencement of frying practices, there could already be a significant degradation of frying oils linked to a concomitant evolution of toxic compounds. Moreover, in view of the lack of governmental regulations, such CFOs can easily be adulterated, and the number of frying cycles is highly dependent 
on user identities [45]. Indeed, during these frying episodes, the end of use of specific CFOs is highly driven by their organoleptic and physical properties, especially the smokepoint and 'smoking' criteria observed, and not their chemical composition and toxic LOP status; such organoleptic properties exert a major impact on consumer perceptions of fried food quality.

Such research investigations [45] have also indicated that apart from the frequent, almost regular over-use of frying oils by particular vendors (i.e., those using repeated frying cycles), there was a clear tendency for them to sell the 'end-of-use oil' to other users, a process which retains the highly degraded oil within circulation for further frying episodes. Consequently, this process potentially endangers the health and lives of many consumers. Furthermore, there remains the possibility that such already-used frying oils could be employed to adulterate fresh frying oils, re-packaged and marketed as fresh, uncontaminated products to unsuspecting consumers, especially in informal population settlements. Hence, these results demonstrated that 'in-use' or pre-discarded CLO samples were of an unacceptable peroxidation status, and therefore may indeed pose adverse health effects to fried food consumers, as demonstrated in [45]. This was corroborated by the total polar compound contents of these oils, which indicated high levels of hydrolytic degradation products (i.e., free FAs, along with mono- and diacylglycerols, and free glycerol) therein [46].

However, it should be noted that many East African sunflower oil products have a somewhat unusual pattern of acylglycerol FAs, i.e., they have higher and lower levels of relatively peroxidation-resistant MUFAs and peroxidation-susceptible PUFAs, respectively, than those of corresponding commercially available, refined Western world oils. Therefore, they appear to be more suitable for frying purposes, prolonged or otherwise [47]. This high oleoylglycerol content characteristic has been postulated to arise from differential geospatial, climatic and altitudinal cultivation conditions, for example [47].

Notwithstanding, many analytical methods available for the determination of such LOPs are either markedly unspecific, with many interfering stages or agents, or remain considerably outdated. However, the multicomponent analytical ability of high-resolution nuclear magnetic resonance (NMR) spectroscopy allows the rapid, virtually non-invasive, nondestructive, and simultaneous study of complex mixtures of agents that are present in edible oil products, and more generally for determining the nutrient status of foods $[12,20,48]$. Further advantages offered are that the technique has no major requirements for detailed knowledge of sample composition prior to analysis (i.e., it is an 'untargeted' technique), and chemical shifts, coupling patterns, and coupling constants of resonances within the ${ }^{1} \mathrm{H}$ and ${ }^{13} \mathrm{C}$ NMR profiles of such samples provide a very high level of confirmatory evidence for the molecular structures of a multitude of lipidic and lipid-soluble agents detectable, including both major and minor analytes. Sample preparation steps involved are very straightforward and rapidly completed.

Hence, to date, high-resolution NMR analysis has been proven to provide a highly valuable, virtually non-destructive high-throughput analytical technique for monitoring the molecular nature and levels of many major acylglycerol FA species, along with a wide range of minor lipid-soluble agents such as chain-breaking antioxidants, phytosterols, terpenes and chlorophylls, in edible oil samples $[12,21,47,49]$. Of especial benefit are the applications of this analytical strategy to determine a series of sequentially generated LOPs, e.g., CHPDs and HPMs, along with a range of aldehydic and further fragmentation products, present in unused or used CFOs [12,20,50]. Furthermore, low-field (60-100 MHz operating frequency) benchtop ${ }^{1} \mathrm{H}$ NMR analysis has been shown to provide a similar sensitivity, and a much-enhanced selectivity, to those offered by Fourier-transform infra-red (FTIR) analysis, which is also utilised for the chemical characterisation of lipid profiles in such samples. Therefore, to date this low-field (LF) NMR analysis approach serves as a valuable screening probe for the rapid, simultaneous analysis of various lipid species in CFOs, and also for purposes of determining the authenticities of such products [51]. 
Primarily, this study further explores the ability of multicomponent high-field (HF) ${ }^{1} \mathrm{H}$ NMR spectroscopic analysis to determine the concentrations of a series of highly toxic aldehydic LOP classes in commonly employed frying oils when exposed to laboratorysimulated shallow frying episodes (LSSFEs) at $180^{\circ} \mathrm{C}$ for increasing durations (0-90 min); oils investigated were PUFA- and PUFA-/MUFA- rich sunflower and canola seed oils, respectively, and MUFA-and antioxidant-rich extra-virgin olive oil. These experiments were performed in order to provide valuable information and insights regarding the possible health-threatening effects (or safety) of aldehyde-containing seed and other edible vegetable oils when ingested in human diets featuring fried food sources of them, e.g., potato chips, fish fillets, beef patties, etc. We have also incorporated SFA-rich coconut oil as a frying medium into these investigations, since only very limited levels of aldehydic LOPs are expected to be generated therein in view of its very low PUFA and MUFA contents. This phase of the study also further investigates the applications of the LF benchtop ${ }^{1} \mathrm{H}$ NMR technique to reliably address the analysis of aldehydic LOPs in such oils when exposed to thermal stressing episodes performed according to the above LSSFE protocol (the first report of this LF NMR analysis application can be found in [11]). Its potential impact on the analysis of commercial CFO products (e.g., direct 'on-site' rapid determinations of triacylglycerol FA compositions and LOP contents) at non-laboratory locations within CFO production centres and restaurants, etc., is discussed.

Subsequently, the above results are reviewed with regard to considerations of the poor reliabilities and specificities of more conventional non-NMR-based spectrophotometric methodologies available for the quantitative determination of dietary LOPs in thermally stressed cooking oils, fried foods, and foods in general. A potential 'cleaner' alternative analytical protocol for one of these frequently employed methods (the TBARS test) is proposed.

Finally, in the light of these results, we outline and discuss the potential adverse toxicological health threats presented by the human ingestion of foods containing significant quantities of pre-heated, used cooking oils. We also critically review reports focused on the negligible or limited health risks offered by PUFA-rich culinary frying oils when employed for frying purposes, and that foods fried therein are 'safe' for human consumption. Contrasting population public health threats potentially posed by the ingestion of dietary LOPs are also explored.

\section{Materials and Methods}

\subsection{Culinary Frying Oils and Fats}

Sunflower, corn, canola, extra-virgin olive and coconut oils were all purchased from local UK retail stores, as was soybean oil and pork lard for the LF benchtop NMR investigations (the sunflower, corn, canola and coconut oils were all refined products). Each oil was then de-identified in the laboratory via its transference to coded but unlabelled storage containers. The specified SFA, MUFA and PUFA contents of these oils were $11.0 \%, 28.0 \%$ and $61.0 \%(w / w)$ for sunflower oil; $14.0 \%, 23.4 \%$ and $62.6 \%(w / w)$ for corn oil; $7.0 \%, 64.4 \%$ and $28.5 \%$ for canola oil; $13.0 \%, 77.4 \%$ and $9.4 \%$ for extra-virgin olive oil; and $90.7 \%, 7.5 \%$ and $1.8 \%(w / w)$, respectively, for coconut oil. The percentage of omega-3 FAs in these samples was estimated by our own previously published ${ }^{1} \mathrm{H}$ NMR method [52], and these contents, predominantly that of linolenic acid, were found to be $0.29 \%, 8.35 \%, 0.73 \%$ and $0.00 \%(w / w)$ for the sunflower, canola, extra-virgin olive and coconut oil products tested, respectively. Moreover, the smoke-points of these oils were $228^{\circ} \mathrm{C}$ for sunflower oil, $234{ }^{\circ} \mathrm{C}$ for corn oil, $226^{\circ} \mathrm{C}$ for canola oil, $195-205^{\circ} \mathrm{C}$ for extra-virgin olive oil, and $203^{\circ} \mathrm{C}$ for coconut oil. Further experiments involved a commercially available sample of refined soybean oil (smoke-point $233{ }^{\circ} \mathrm{C}$ ); its acylglycerol FA composition was 15.3, 25.3 and 59.4 molar \% for SFAs, MUFAs and PUFAs, respectively, the latter including 9.3 molar \% linolenic acid. A locally purchased pork lard (tallow) sample was found to contain 46.6, 47.5 and 5.9 molar $\%$ SFAs, MUFAs and PUFAs, respectively. The molar \% contents of total UFAs, PUFAs, MUFAs and SFAs of these oils were estimated by modifications of previously reported ${ }^{1} \mathrm{H}$ 
NMR methods that are outlined in Ref. [22]. This involved a consideration of the intensities of integrated intelligently selected ${ }^{1} \mathrm{H}$ NMR buckets containing their acylglycerol bis-allylic$\mathrm{CH}=\mathrm{CH}-\mathrm{CH}_{2}-\mathrm{CH}=\mathrm{CH}-,-\mathrm{CH}_{2}-\mathrm{CH}=\mathrm{CH}-, \alpha-\mathrm{CH}_{2}-\mathrm{CO}_{2}-$ and glyceryl backbone- $\underline{\mathrm{H}}_{2} \mathrm{OCOR}$ function resonances $(\delta=2.73-2.88,1.96-2.13,2.26-2.37$ and $4.10-4.15 / 4.28-4.33$ ppm, respectively), and the use of equations specified for the estimation of these contents. ${ }^{1} \mathrm{H}$ NMR-determined molar \% FA contents obtained were in very good agreement with those specified by the manufacturers (i.e., within \pm 0.25 molar \% of the values provided above).

\subsection{Laboratory-Simulated Shallow Frying Episodes}

All oils were exposed to thermal stressing episodes at $180{ }^{\circ} \mathrm{C}$ for periods of up to 90 min according to an established laboratory-simulated shallow frying episode (LSSFE) protocol [13], and these experiments were conducted by a 'blinded' laboratory researcher. Each 90 min heating cycle was conducted $n=6$ replicated times for all oils investigated. LSSFEs involved the heating of a $6.00 \mathrm{~mL}$ volume of culinary oil in a $250 \mathrm{~mL}$ thoroughly airdried glass beaker maintained within a silicon oil bath accurately regulated at a temperature of $180^{\circ} \mathrm{C}$ throughout the total $90 \mathrm{~min}$ heating period employed. Aliquots (ca. $0.25 \mathrm{~mL}$ ) of oil samples were collected at the $0,5,10,20,30,60$ and 90 min heating time-points for ${ }^{1} \mathrm{H}$ NMR analysis. Immediately following collection, the lipid-soluble antioxidant 2,5-di-tert-butylhydroquinone (DTBHQ) was added to each oil sample at a final added concentration of $10.00 \mathrm{mmol} \mathrm{kg} \mathrm{m}^{-1}$ in order to block or retard the further generation of aldehydes and their HPM and CHPD precursors during periods of storage, and sample preparation at ambient temperature. Samples were prepared for ${ }^{1} \mathrm{H}$ NMR analysis within $2 \mathrm{~h}$. after collection, and were stored in sealed containers within a light-excluded zone at $4{ }^{\circ} \mathrm{C}$ whilst awaiting analysis.

\section{3. ${ }^{1} \mathrm{H}$ NMR Analysis}

High-resolution ${ }^{1} \mathrm{H}$ NMR spectroscopic analysis of ca. $3 \times$ dilutions of the sampled oils in deuterochloroform $\left(\mathrm{C}^{2} \mathrm{HCl}_{3}\right)$ was performed on Bruker Avance AM-400 or JEOLECZR600 NMR spectrometers (De Montfort University, Leicester, UK facilities) operating at frequencies of 400.13 and $600.17 \mathrm{MHz}$, respectively, and a probe temperature of $298 \mathrm{~K}$, as previously described $[19,20]$. Selective quantification of CFO aldehyde classifications ( $\mathrm{mmol} / \mathrm{kg}$ or $\mathrm{mmol} / \mathrm{mol} \mathrm{FA}$ ) was performed via the electronic integration of 9 specified aldehyde-C $\underline{\mathrm{HO}}\left(\delta=9.40-10.20 \mathrm{ppm}\right.$ range), acylglycerol chain terminal- $\mathrm{CH}_{3}$ function $(\delta=0.88-0.99 \mathrm{ppm}$ in total) and/or pre-added internal standard proton resonances, as outlined in $[12,13]$. Calibration curves for aldehyde standard solutions in $\mathrm{C}^{2} \mathrm{HCl}_{3}$, and those 'spiked' into selected unheated aldehyde-free CFO samples (0.03-60 mmol per $\mathrm{L}$ and $\mathrm{kg}$, respectively), were linear ( $\mathrm{r}=0.990-0.998)$. 'Between-frying cycle' sample coefficients of variation for aldehyde classification determinations were computed from the $n=6$ replicated thermal stressing episodes, and these ranged from $3.7-12.3 \%$ for all oils investigated. Those for intra-assay repeat determinations made on the same samples were $\leq 2.5 \%$.

LF (60 MHz) ${ }^{1} \mathrm{H}$ NMR spectra of both baseline control and LSSFE-exposed, heated oil or lard samples prepared in $\mathrm{C}^{2} \mathrm{HCl}_{3}$ solution as outlined above were obtained on a Magritek Spinsolve benchtop spectrometer operating at a frequency of $61.67 \mathrm{MHz}$. Spectra were acquired using a 1D Proton+ sequence. The parameters utilised for these analyses were: $32 \mathrm{~K}$ data points; 128 scans; acquisition time $6.4 \mathrm{~s}$; a repetition rate $10 \mathrm{~s}$; pulse angle $90^{\circ}$.

\subsection{Specific Spectrophotometric Method for the Analysis of Malondialdehyde}

Malondialdehyde (MDA) concentrations in edible CFOs were specifically determined by a modification of the method described by Claxson et al. (1994) [19], which involved the reaction of thiobarbituric acid (TBA) with this dialdehyde to form a pink-red chromophore following its extraction into an aqueous medium. This method is interference-free, since 
the 470-600 nm regions of electronic absorption spectra of such aqueous extracts contain absorption bands arising from the 2:1 TBA:MDA adduct only $\left(\lambda_{\max } 532 \mathrm{~nm}\right)$.

\subsection{Statistical Analysis of Patterns of Aldehydic LOPs Generated during LSSFEs}

For sunflower, canola, extra-virgin olive and coconut oils heated according to LSSFEs, boxplots of median $\pm 95 \%$ confidence interval $(\mathrm{CI})$ values were plotted for the concentrations of trans-2-alkenals, alka-(trans,trans)-2,4-dienals and $n$-alkanals generated at each of the $0,5,10,20,30,60$ and 90 min time-points ( $n=6$ replicates per oil per time-point). The $95 \%$ CIs for the median aldehyde levels were calculated as $\pm(1.58 \times I \mathrm{IQR}) /(\mathrm{n})^{1 / 2}$, where IQR represents the inter-quartile range (i.e., a statistical dispersion measure equivalent to the difference between the distributional upper and lower quartiles), and $n=6$.

The significance of differences found between culinary oils and LSSFE heating timepoints, and also that for the culinary oil $\mathrm{x}$ time-point interaction effect, was determined by an ANOVA model with the experimental design shown in Equation (1). In this equation, $\mathrm{O}$ and $\mathrm{T}$ represent the main oil class and time-point factors, and OT the first-order oil $\mathrm{x}$ time-point interaction effect, which was expected to be significant in view of differential oil-dependent levels of aldehydes generated at increasing time-points, i.e., the pattern of aldehyde level responses differed for each oil evaluated; $\mu$ denotes the null hypothesis mean value in the absence of any sources of variation, and $\mathrm{e}_{i j k}$ fundamental error.

LSSFE time-points were considered as qualitative variables in an ANOVA model rather than quantitative ones in an ANCOVA one in view of the non-linearity of plots of mean aldehyde concentrations versus time-point (Figure 2). Indeed, these are predominantly sigmoidal (S-shaped) curves in view of the self-propagating autocatalytic nature of the lipid peroxidation process.

$$
\mathrm{y}_{i j k}=\mu+\mathrm{O}_{i}+\mathrm{T}_{j}+\mathrm{OT}_{i j}+\mathrm{e}_{i j k}
$$

Agglomerative hierarchical clustering (AHC) analyses of aldehydic LOP datasets was performed using XLSTAT2016 and Metaboanalyst 5.0 software module options. Two AHC strategies were employed for analysing the aldehydic LOP dataset: The first model considered only the 3 major aldehydes generated, and data were generalized logarithmically (glog)-transformed and autoscaled prior to analysis; for the second model, which considered 7 aldehydic LOPs, data were autoscaled only. Autoscaling involves subtraction of the aldehyde class (column) variable from all observations in that column, followed by division by the column standard deviation, so that all aldehyde class variables have a mean value of zero and a variance (and standard deviation) of unity. AHC dendograms were generated using a Euclidean distance measure, and Ward's linkage clustering algorithm.

\section{Results and Discussion}

\subsection{HF ${ }^{1}$ H NMR Case Study: Time-Dependent Tracking of Aldehydic LOPs in CFOs Exposed to LSSFES}

In the first phase of the current study, the wide range of analytical advantages offered by high-resolution ${ }^{1} \mathrm{H}$ NMR technologies for the analysis of LOPs in used or unused frying oils is highlighted: this is presented as a case study involving the heating of a series of four CFO products with differing acylglycerol contents and degrees of unsaturation, according to laboratory-simulated shallow frying episodes (LSSFEs) conducted at a recommended frying temperature $\left(180^{\circ} \mathrm{C}\right)$.

The levels of individual aldehyde classifications, and total aldehydes, formed in CFOs exposed to the LSSFEs described increased with increasing oil PUFA contents, and also in a sigmoidal time-dependent fashion. For sunflower and canola oils, $>80 \%$ of these were of the more highly toxic $\alpha, \beta$-unsaturated class, which included acrolein, trans-2alkenals [(E)-2-alkenals], alka-trans, trans- and alka-(cis,trans)-2,4-dienals [alka-(E,E)- and alka-(Z,E)-2,4-dienals, respectively], together with combined 4-hydroperoxy/4-hydroxy-, and 4,5-epoxy-trans-2-alkenals (Figures 1 and 2). Moreover, relatively low concentrations of these aldehydes and their CHPD precursors were also detectable in newly purchased, 
unheated corn and sunflower oil products (Figure $1 b, c$, respectively). Primary lag-phases for aldehyde generation, which increased with increasing SFA and MUFA, and decreasing oil PUFA contents, also decreased with increasing peroxidative susceptibilities of the oils tested, as expected [53], which were calculated to be 62.6, 38.5, 12.0 and only 2.0 for the sunflower, canola, extra-virgin olive and coconut oil products tested, respectively. An intermediate lag-phase was also observed at a heating time-point of ca. 20-30 $\mathrm{min}$ for MUFA-rich oils, particularly canola oil. For PUFA-rich sunflower oil, which generated the highest ${ }^{1} \mathrm{H}$ NMR-detectable levels of both $\alpha, \beta$-unsaturated and saturated aldehydes, the maximal mean concentrations of trans-2-alkenals, alka-(trans,trans)-2,4-dienals and $n$-alkanals remaining in this frying medium at the extreme $90 \mathrm{~min}$ heating time-point were ca. 23, 11 and $10 \mathrm{mmol} \mathrm{kg}^{-1}$, respectively: total mean unsaturated and saturated aldehyde levels determined in this heated oil were 47.1 and $10.0 \mathrm{mmol} \mathrm{kg}^{-1}$, respectively. Corresponding mean $(n=6) 90$ min time-point estimates of total unsaturated and saturated aldehydes in the other oils tested were 39.9 and 8.2 (canola oil), 26.1 and 6.9 (extra-virgin olive oil), and only 7.5 and $2.6 \mathrm{mmol} \mathrm{kg}^{-1}$ (coconut oil), respectively.

Of particular interest is the ${ }^{1} \mathrm{H}$ NMR detection of HNE and HHE in thermally stressed culinary oils - these neurotoxic and potentially carcinogenic secondary LOPs are derived from chemical reduction of their corresponding 4-hydroperoxy-trans-2-alkenal precursors. However, since they predominantly arise from the peroxidation of linoleoyl- and linolenoylglycerols, respectively [22], little or none of them are detectable in thermally stressed olive oil products (or other PUFA-deplete oils), since such frying media contain only $5-10 \%$ and $\leq 1 \%(w / w)$ of these triacylglycerol species, respectively. This also serves to explain why much lower levels of similarly toxic alka-(trans,trans)-2,4-dienals are generated in such MUFA-rich oils when exposed to thermal stressing episodes; this class of $\alpha, \beta$-unsaturated aldehydes is only generated from PUFA-derived CHPD species, predominantly decatrans,trans-2,4-dienal from linoleate hydroperoxide sources [11,12,19,20,22].

CANOLA

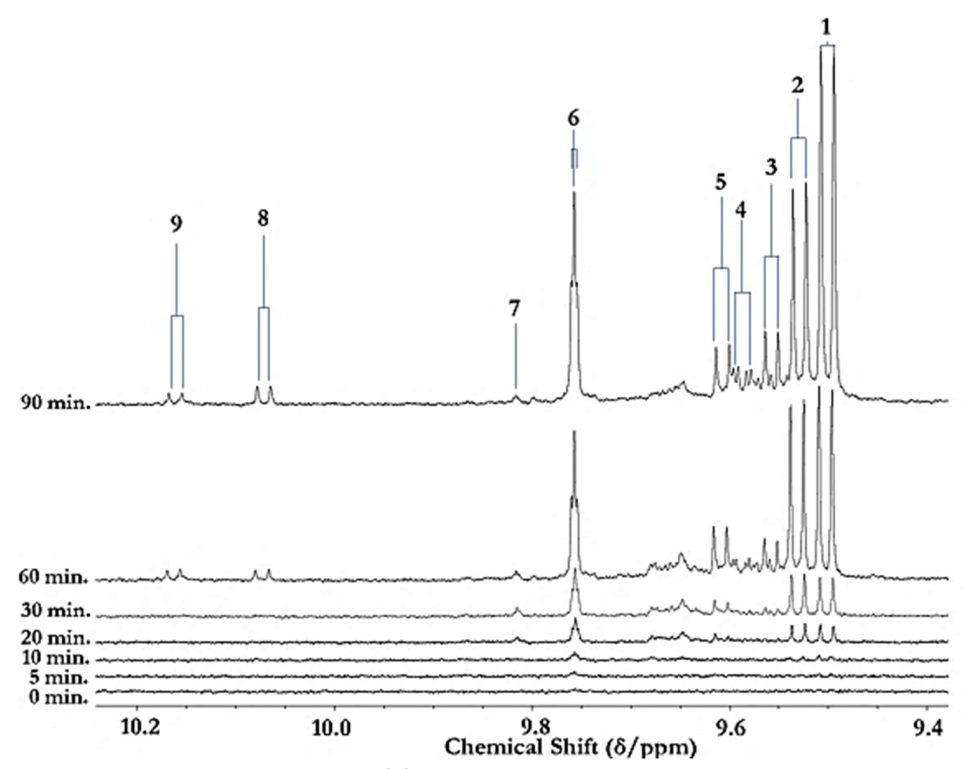

(a)

Figure 1. Cont. 
CORN
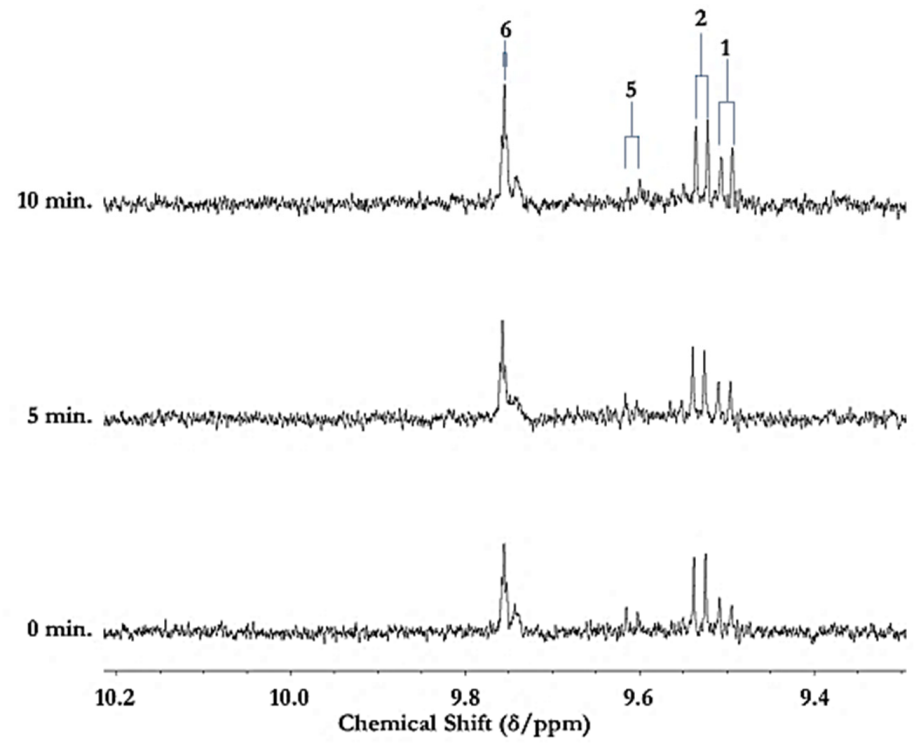

(b)

SUNFLOWER
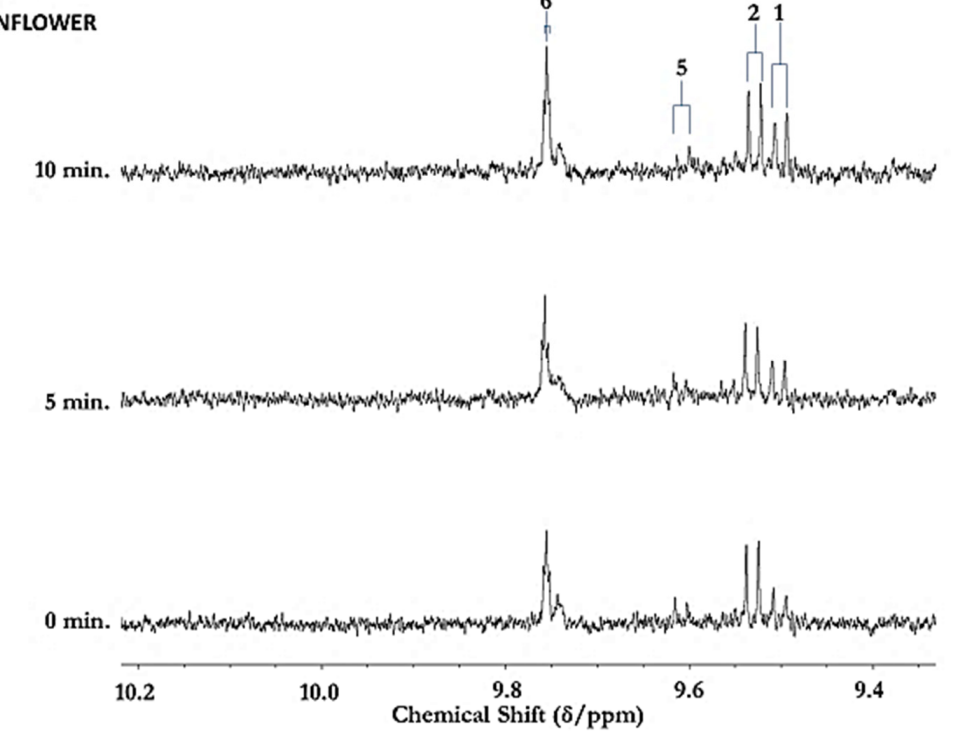

(c)

Figure 1. (a) Expanded aldehydic-CHO proton (9.35-10.20 ppm) region of $600 \mathrm{MHz}{ }^{1} \mathrm{H}$ NMR spectra of a typical canola oil product exposed to thermal stressing episodes at $180^{\circ} \mathrm{C}$ for periods of $0-90 \mathrm{~min}$ according to our LSSFEs (samples were collected for ${ }^{1} \mathrm{H}$ NMR analysis at the 0 (unheated), 5, 10, 20, 30, 60 and 90 min time-points). (b,c), Corresponding $400 \mathrm{MHz}{ }^{1} \mathrm{H}$ NMR spectral regions of commercially available UK brands of corn and sunflower oils, respectively, heated under the same conditions for only 0,5 and $10 \mathrm{~min}$. All resonances visible are doublets $(J=7.63 \mathrm{~Hz}$ for trans-2-alkenals), with the exception of the $n$-alkanal one which is a triplet $(J=1.83 \mathrm{~Hz})$. Assignments for these signals were confirmed via the acquisition of corresponding one- and two-dimensional correlation (COSY) and total correlation (TOCSY) spectra for each heated oil as described in $[12,19,20]$. Detectable aldehyde levels for the unheated (time-point $=0 \mathrm{~min}$ ) corn and sunflower oil samples are notable. Abbreviations: 1, trans-2-alkenals (doublet); 2, alka-(trans,trans)-2,4-dienals (doublet); 3,4,5-Epoxy-trans-2-alkenals (doublet); 4,4-hydroxy-/4-hydroperoxy-trans-2-alkenals (overlapping doublets); 5, alka-(cis,trans)-2,4dienals (doublet); 6 , higher homologue $n$-alkanals (triplet); 7 , low-molecular-mass $n$-alkanals (triplet) such as ethanal and propanal, which predominantly arise from the peroxidation of linolenoylglycerol substrates available in soybean oil (up to $9.5 \%(w / w)$ ); ethanal is also generated from the thermal degradation of deca-2,4-dienals [13]); 8, cis-2-alkenals (doublet); 9, unidentified aldehyde, presumably a substituted cis-2-alkenal species. 
(a) Sunflower Oil

(E)-2-alkenals

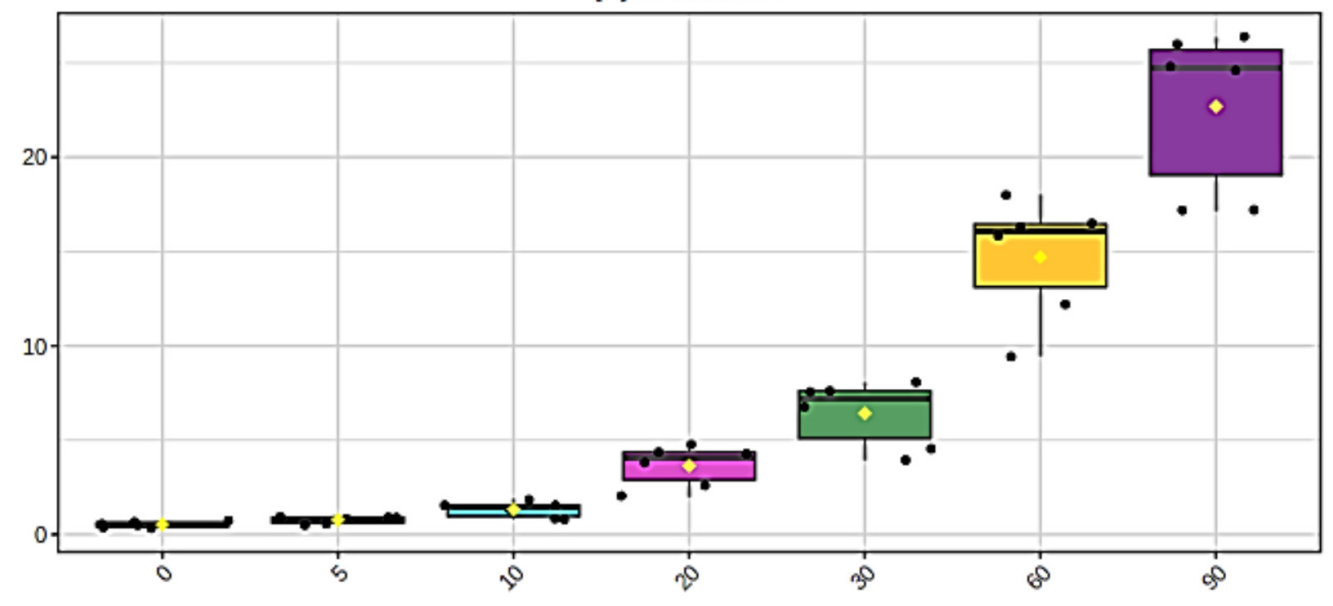

(E,E)-2,4-alkadienals

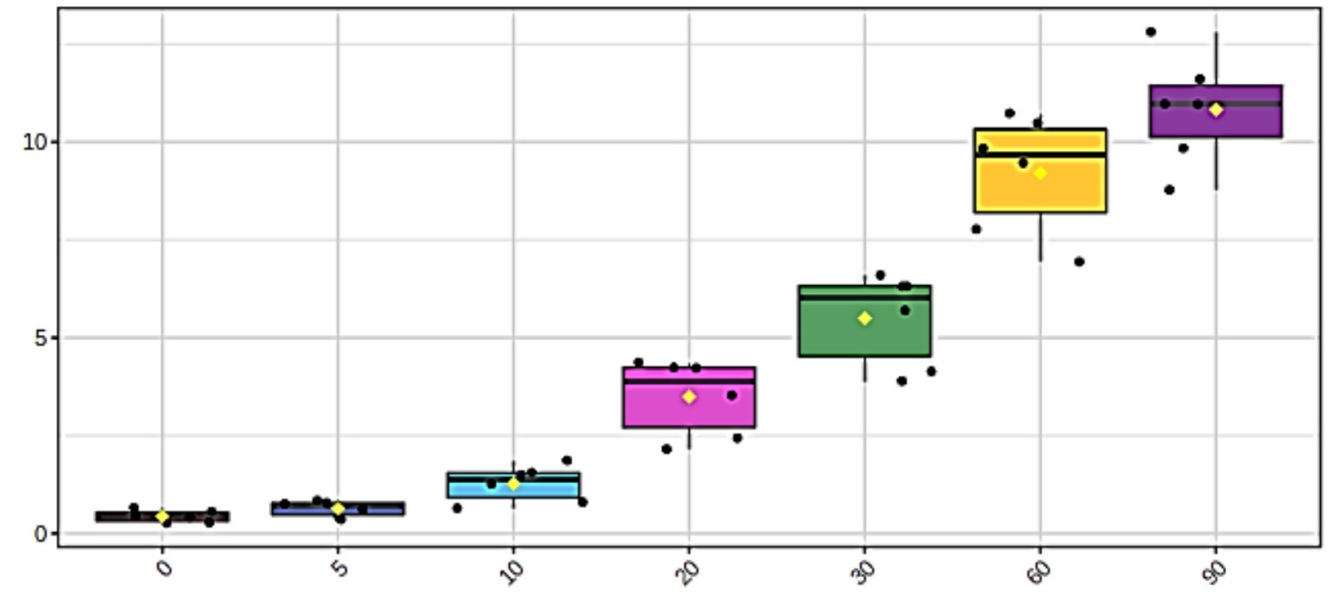

n-alkanals

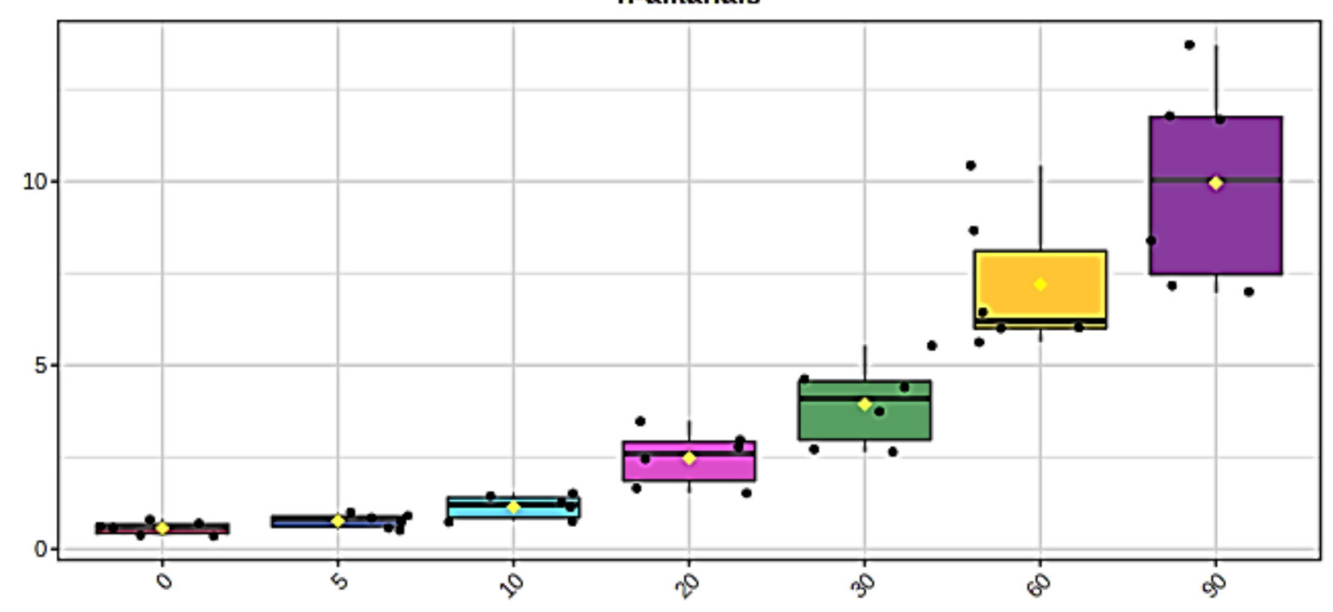

Figure 2. Cont. 


\section{(b) Extra-Virgin Olive Oil}

(E)-2-alkenals

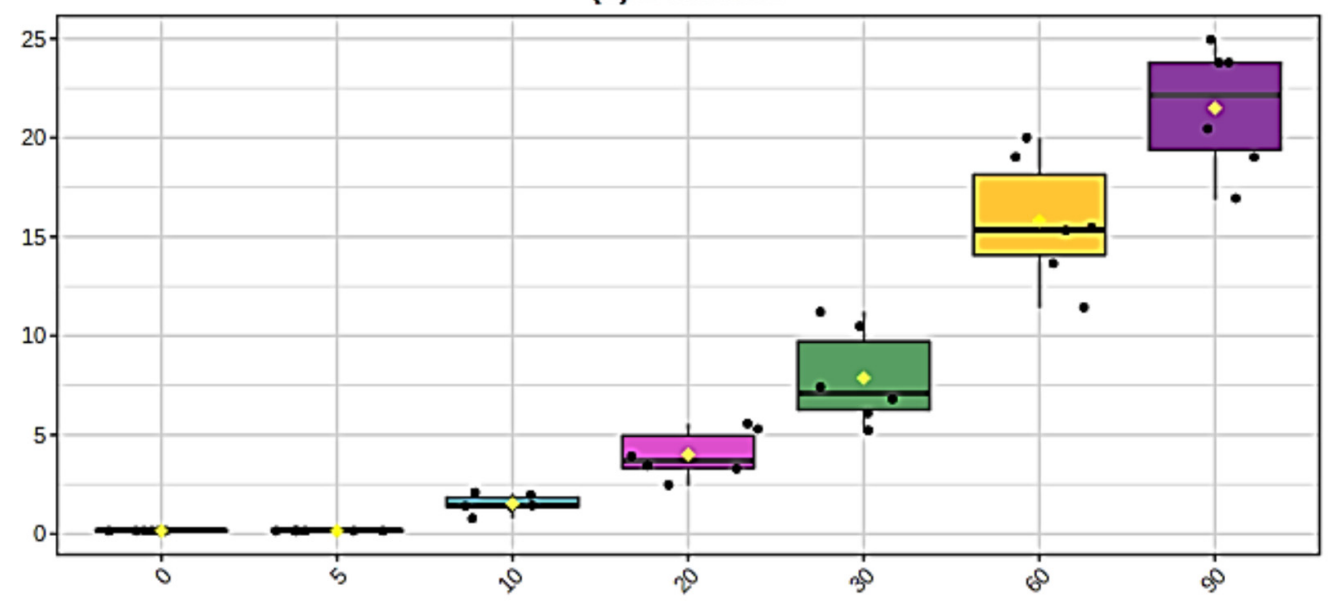

(E,E)-2,4-alkadienals

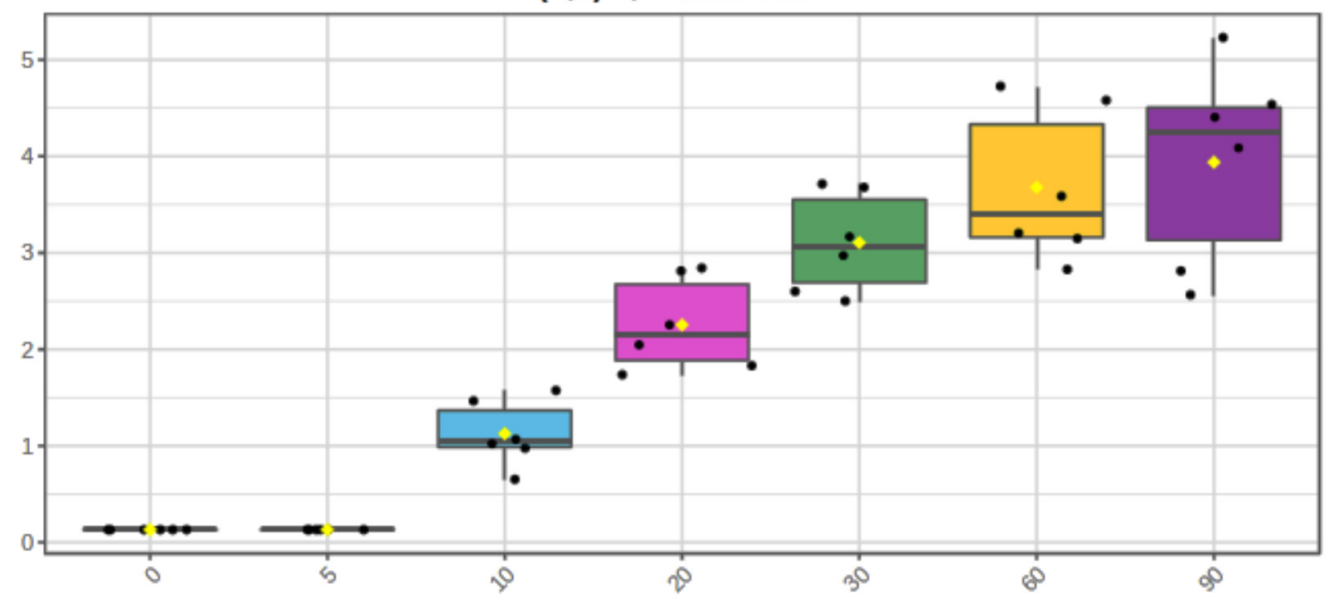

n-alkanals

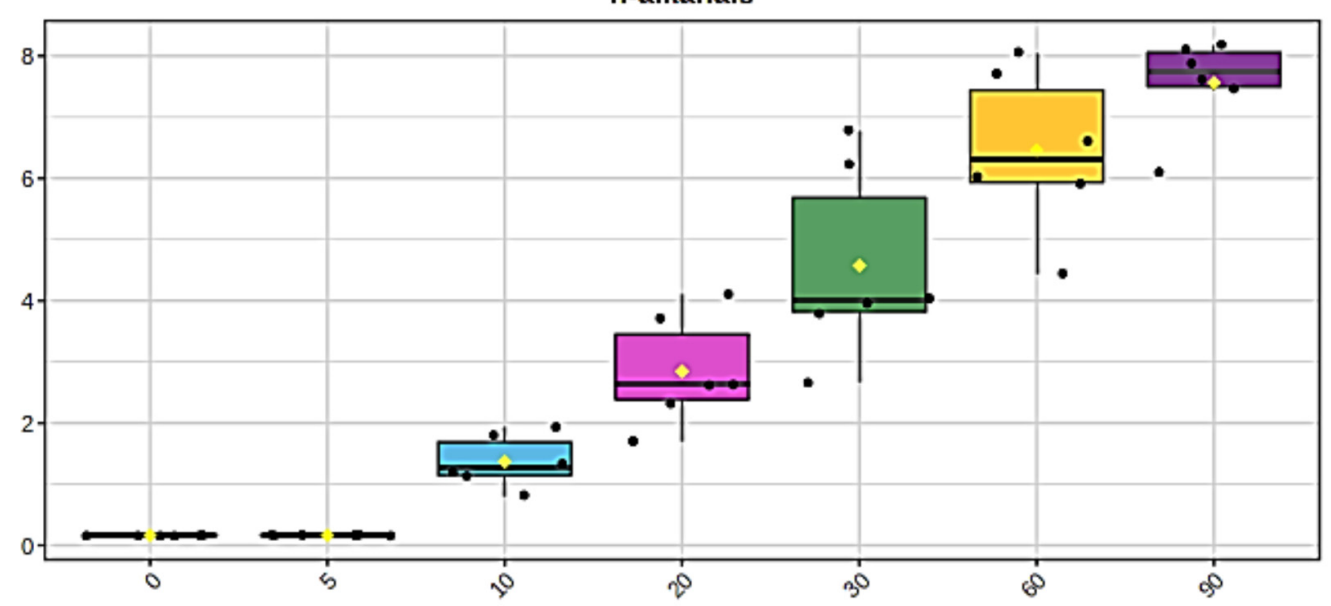

Figure 2. Cont. 


\section{(c) Canola Oil}

(E)-2-alkenals

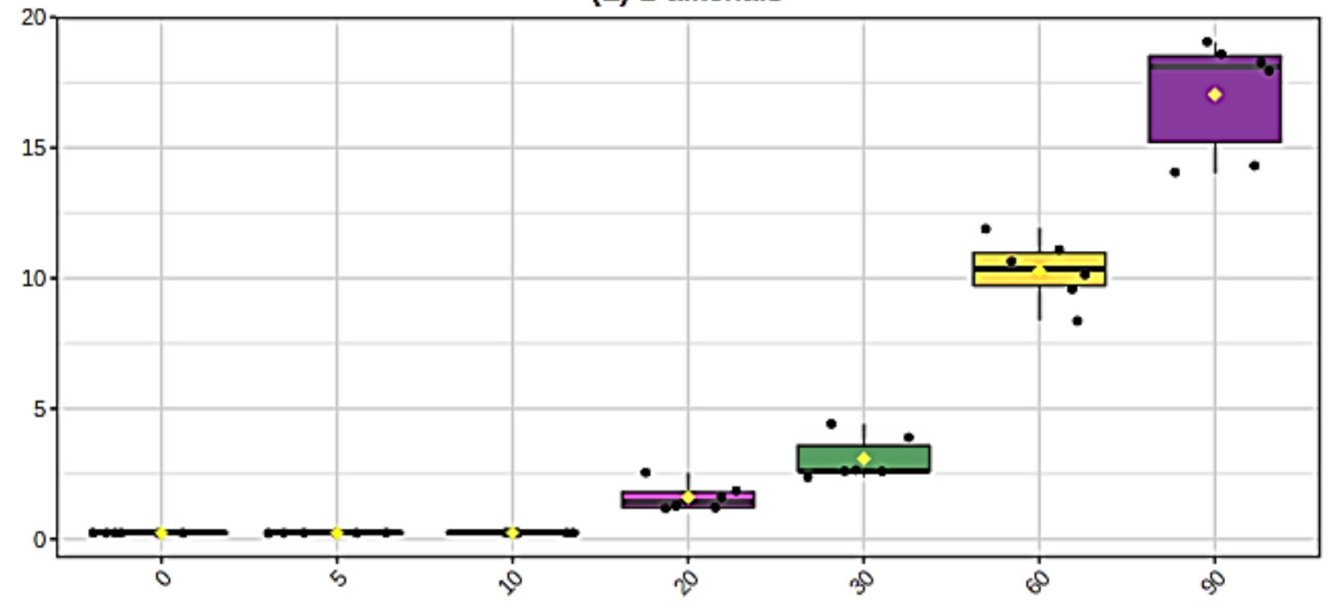

(E,E)-2,4-alkadienals
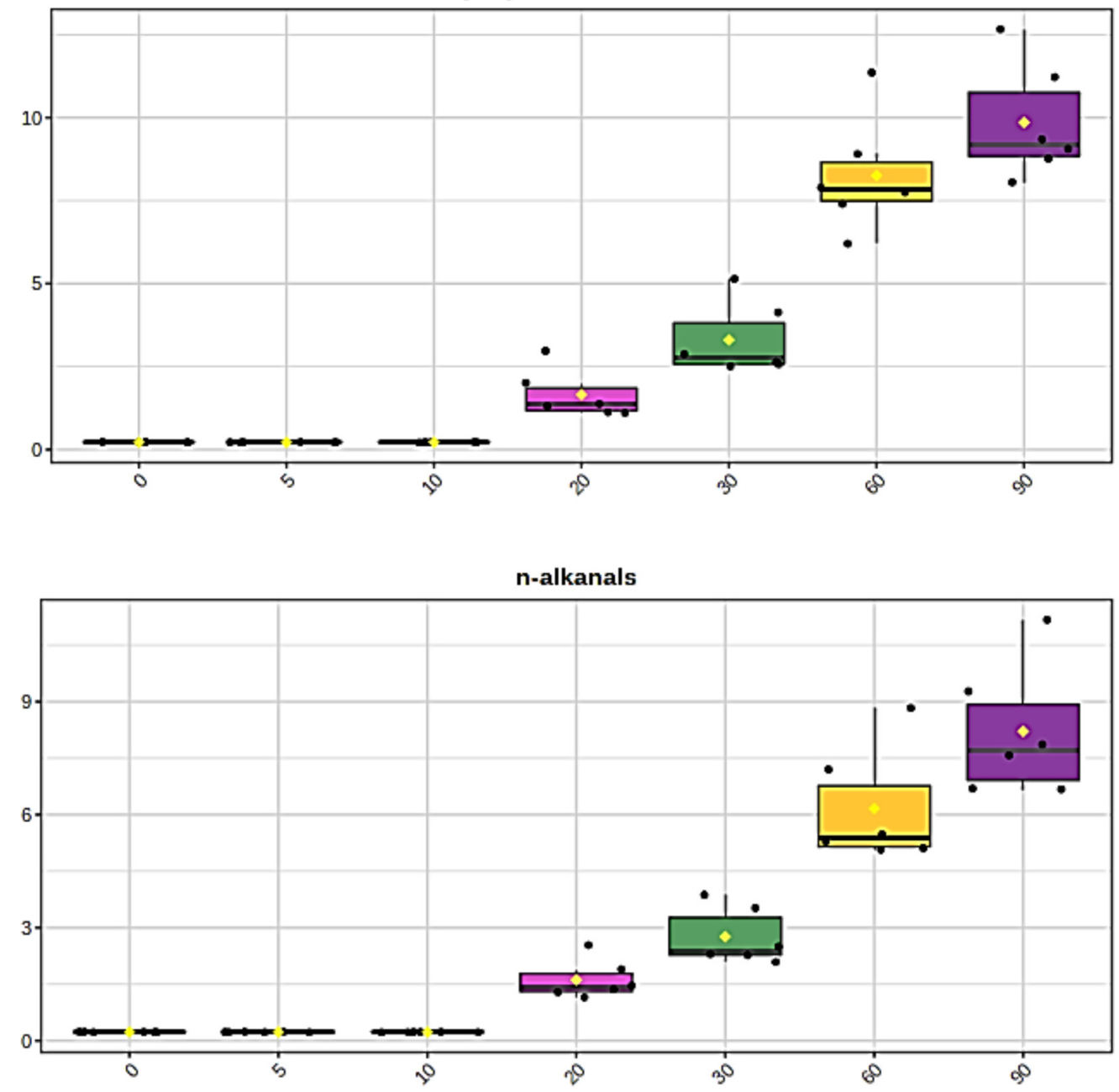

Figure 2. Cont. 
(d) Coconut Oil

(E)-2-alkenals

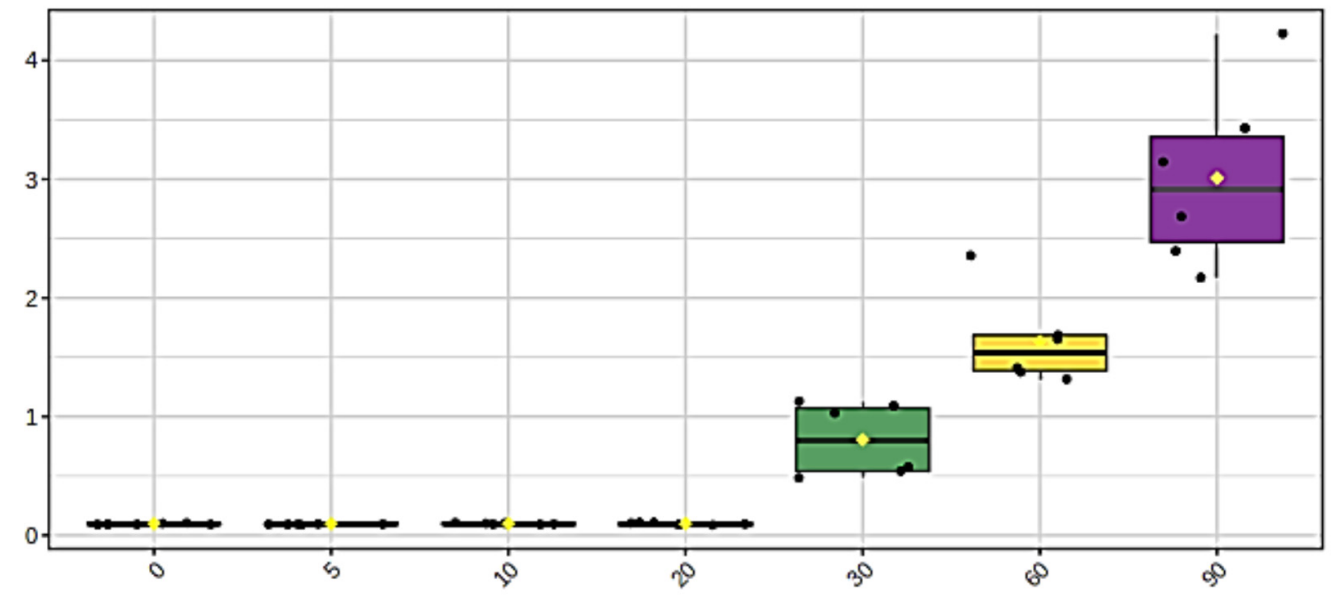

(E,E)-2,4-alkadienals

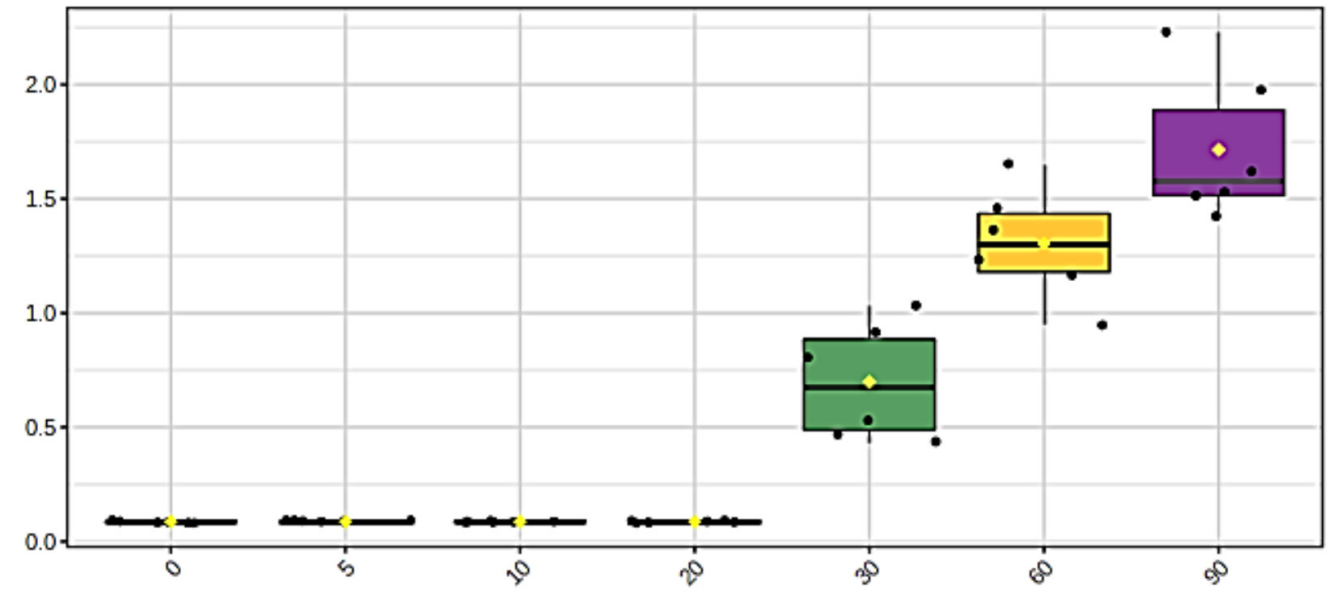

n-alkanals

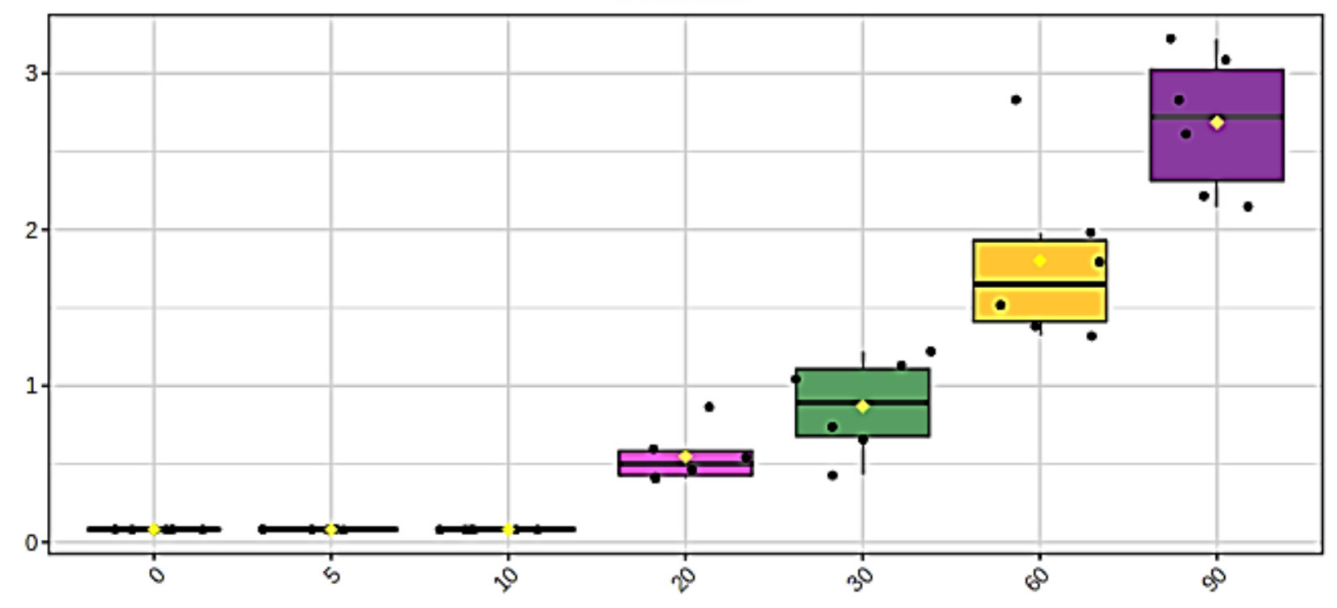

Figure 2. Cont. 

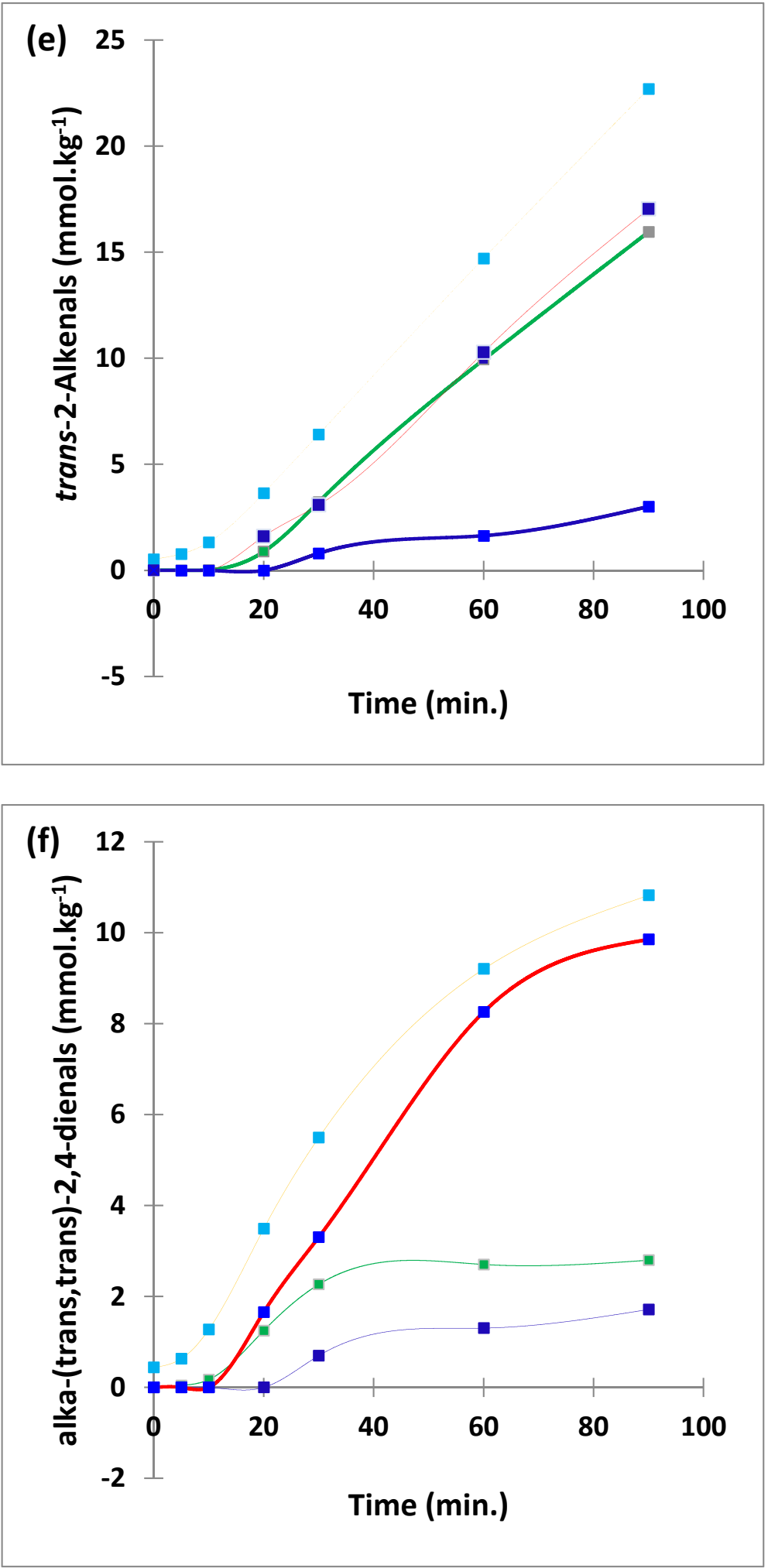

Figure 2. Cont. 


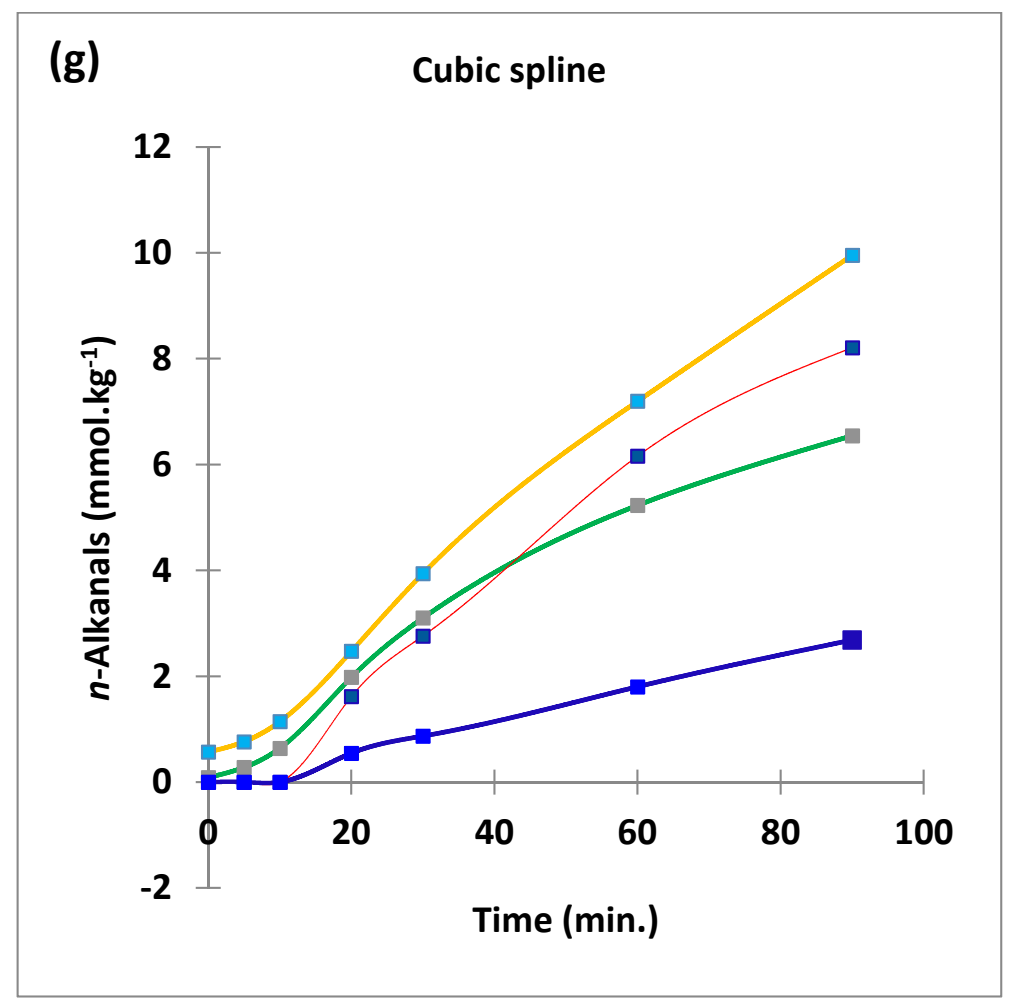

Figure 2. Boxplots of median $\pm 95 \%$ confidence intervals (CIs) levels of ${ }^{1} \mathrm{H}$ NMR-determined trans2-alkenals, alka-(trans,trans)-2,4-dienals and $n$-alkanals generated in (a) sunflower, (b) extra-virgin olive, (c) canola and (d) coconut oils at 0, 5, 10, 20, 30, 60 and $90 \mathrm{~min}$ time-points of exposure to LSSFEs at a temperature of $180^{\circ} \mathrm{C}$. The notch depicts the median CIs, and the mean concentrations of each group are indicated by yellow diamonds. Abscissa and ordinate axes are heating timepoints and aldehyde concentrations $\left(\mathrm{mmol} \mathrm{kg}^{-1}\right)$, respectively. $(\mathbf{e}-\mathbf{g})$, Corresponding cubic spline polynomial plots of mean total ${ }^{1} \mathrm{H}$ NMR-determined trans-2-alkenal, alka-(trans,trans)-2,4-dienal and $n$-alkanal concentrations, respectively, as a function of heating time for coconut (blue), canola (red), extra-virgin olive (green) and sunflower (yellow curve) oils. The cubic spline analyses performed on our time-dependent aldehyde concentration datasets involved a piecewise function of thirddegree polynomials; computation of the cubic spline coefficients featured polynomial derivatives [54]. Results from univariate statistical analysis performed by an ANOVA model (Equation (1)) for the 'between-oils', 'between-time-points' and oils $x$ time-points interaction effects were $p=2.49 \times 10^{-44}$, $1.09 \times 10^{-82}$ and $1.17 \times 10^{-38}$, respectively for trans-2-alkenals; $p=5.98 \times 10^{-50}, 1.39 \times 10^{-74}$ and $2.23 \times 10^{-41}$, respectively for alka-trans,trans-2,4-dienals; and $p=5.10 \times 10^{-28}, 1.15 \times 10^{-64}$ and $1.04 \times 10^{-16}$, respectively for $n$-alkanals. The highly significant interaction effects observed for all aldehydes indicate the differential time dependencies of their concentrations generated for each oil investigated.

The mean concentration magnitude orders of aldehyde generation at typical shallowfrying sampling time-points of only 10 and $20 \mathrm{~min}$ (the latter commonly employed for the frying of chicken strips, for example) were found to be sunflower $>>>$ canola $\approx$ extra-virgin $\approx$ coconut oils (non- ${ }^{1} \mathrm{H}$ NMR detectable in the latter three), and sunflower $>$ canola $>$ extravirgin olive $\approx$ coconut oils (undetectable in the latter two), respectively, for trans-2-alkenals; sunflower $>$ extra-virgin olive $>$ canola $\approx$ coconut oils (undetectable in the latter two), and sunflower $>$ extra-virgin olive $>$ canola $>$ coconut oils (undetectable in the latter medium only), respectively, for $n$-alkanals. However, for peroxidised linoleoylglycerol-derived alka-(trans,trans)-2,4-dienals, these orders were sunflower $>>$ extra-virgin olive $>$ canola $\approx$ coconut oils (undetectable in the latter two), and SFO $>>$ canola $>$ extra-virgin olive $>$ coconut oils (undetectable in the latter oil only). At the 5 min shallow frying-simulated time- 
point, however, all three of these aldehydes were only detectable in PUFA-rich sunflower and corn oils. Mean concentrations of total trans-2-alkenals, alka-(trans,trans)-2,4-dienals and $n$-alkanals in the PUFA-rich sunflower oil product examined were 3.23, 3.14 and $2.04 \mathrm{mmol} \mathrm{kg}^{-1}$, respectively at the $20 \mathrm{~min}$ LSSFE time-point.

${ }^{1} \mathrm{H}$ NMR spectra of heated sunflower and canola oils also contained signals assignable to aldehydic precursors, in particular cis,trans- and trans,trans-CHPDs (multiplet resonances located within the 5.40-6.60 and 5.40-6.30 ppm spectral regions, respectively, together with broad -OOH function signals centred at $\delta=8.40-8.85 \mathrm{ppm}$ ); and cis, trans-conjugated hydroxydienes $(\delta=5.40-6.50 \mathrm{ppm}$ range), as previously reported $[12,19,20]$ (data not shown).

\subsection{AHC Analysis of Aldehydic LOP Variables}

Agglomerative hierarchal clustering (AHC) analysis of only the three major aldehydic LOP variables (Figure 3) indicated that total trans-2-alkenal and alka-(trans,trans)-2,4dienal levels were correlated; these aldehydes are derived from the peroxidation of PUFAs (linoleoyl- and linolenoylglycerols), the latter from these acylglycerol FA sources alone. Additionally, 2-heptenal isomers arise from the degradation of alka-2,4-decadienals, together with acetaldehyde, hexanal, acrolein, butenal, 2-heptenal and 2-octenal, amongst other aldehydes [55].

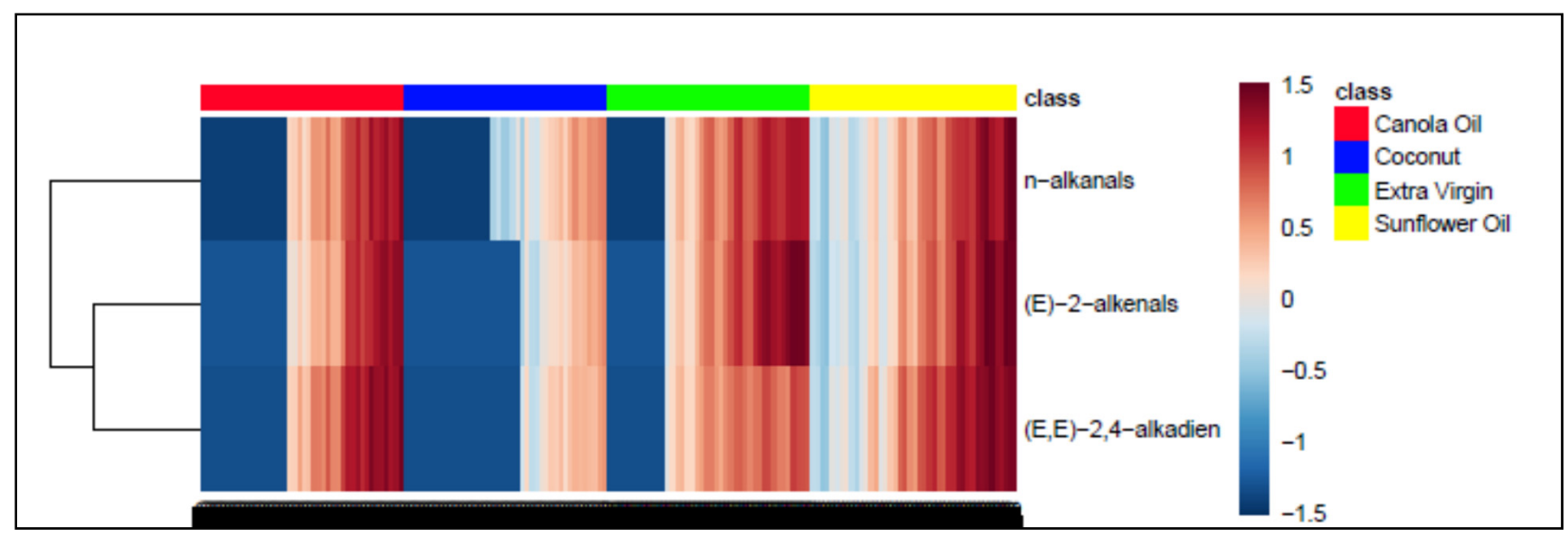

Figure 3. AHC monoclustering heatmap diagram displaying, from left to right, increasing concentrations of the three major aldehydic LOPs ( $n$-alkanals, trans-2-alkenals and alka-trans,trans-2,4-dienals) as a function of LSSFE time-point for canola (red), coconut (blue), extra-virgin (green) and sunflower oils (yellow); there were $\mathrm{n}=6$ replicate samples analysed per time-point. The dataset was glog-transformed and autoscaled prior to analysis. Transformed aldehyde concentrations are shown in the right-hand side $y$-axis: deep blue and red colourations represent extremes of low and high levels, respectively. The left-hand side of the plot shows results arising from a preliminary AHC analysis of these aldehydic LOP variables monitored. Abbreviation: (E,E)-2,4-alkadien represents alka-(trans,trans)-2,4-dienals.

This AHC analysis also indicated that these correlated aldehyde concentrations were distinguishable from that of total $n$-alkanals (data not shown), which are known to arise from the peroxidation of MUFAs as well as PUFAs. However, the substitutional status of linoleate, linolenate and perhaps further PUFAs at the glycerol backbone of triacylglycerols [i.e., $s n-1(3)$ and /or -2 positions] is also a key determinant of the nature and concentrations of secondary aldehydic LOPs arising from their autoxidation [17].

However, performance of a related AHC analysis of all seven aldehydic LOP variables provided further details regarding the clustering of aldehydic LOPs (Figure 4). Indeed, two major clusters were notable, the first (cluster A) containing more structurally complex aldehyde classes (4,5-epoxy-trans- and 4-hydroxy-/4-hydroperoxy-trans-2-alkenals, along with alka-(cis,trans)- and alka-(trans,trans)-2,4-dienals), while the second comprised more structurally simpler aldehydes, specifically cis- and trans-2-alkenals, and $n$-alkanals (cluster B). From the dissimilarity truncation limit indicated (dotted line in Figure 4), the 
second major cluster was split into two statistically significant sub-clusters (B1 and B2), the first containing cis-2-alkenals alone, the second a trans-2-alkenal and $n$-alkanal combination. Although below the dissimilarity threshold value and therefore not significant, cluster A was further split into two sub-clusters, the first (A1) featuring a combination of 4.5-epoxy-trans-2-alkenals and 4-hydroxy-/4-hydroperoxy-trans-2-alkenals, the second (A2) both alka-(cis,trans)- and alka-(trans,trans)-2,4-dienals.

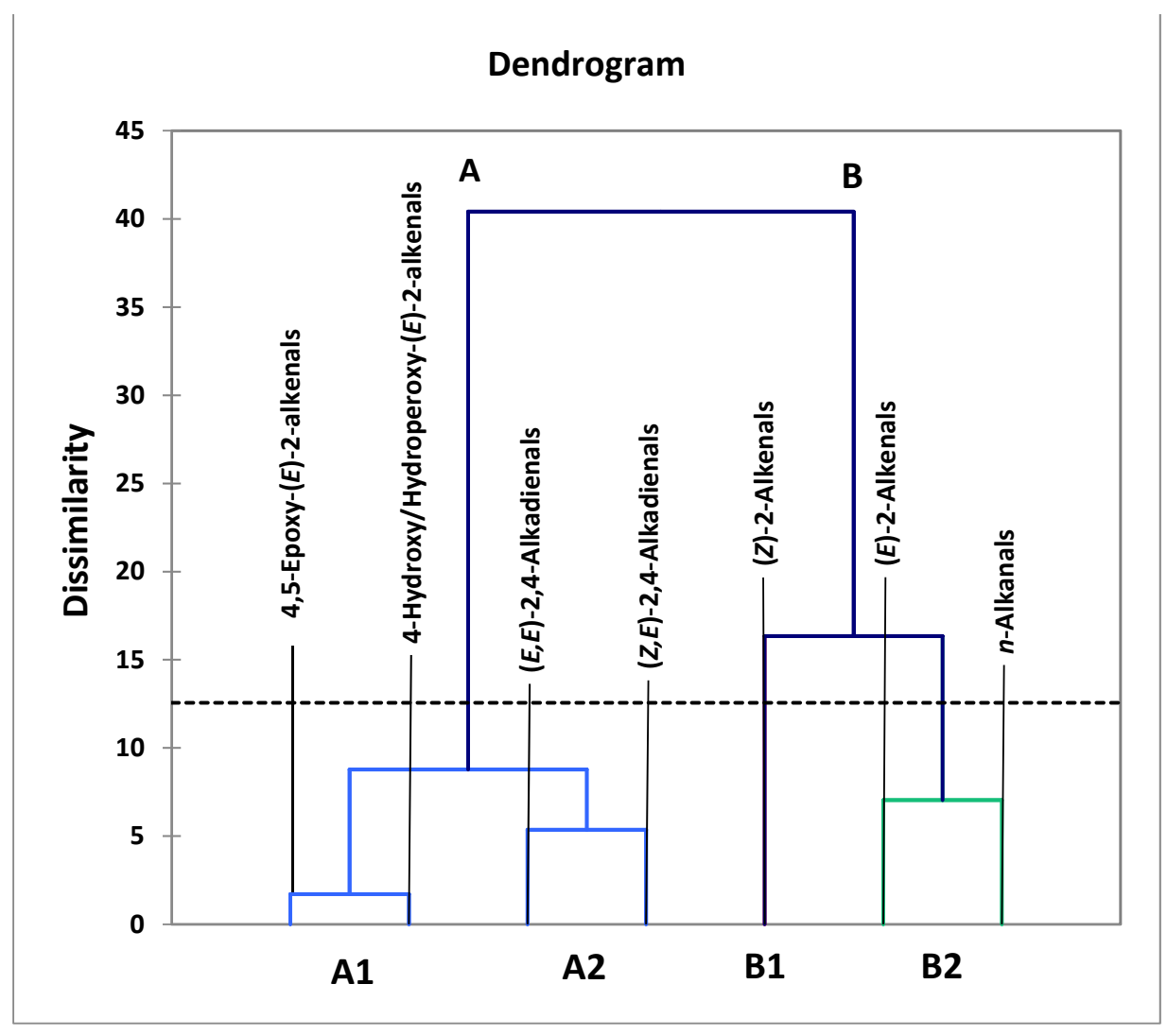

Figure 4. AHC clustering analysis of a model dataset containing 7 aldehyde classification variables, the concentrations of which were those generated at all 7 LSSFE heating time-points. Two major clusters were isolated, the first containing structurally complex aldehydes arising from PUFA peroxidation (cluster A), the second structurally simpler ones generated from both MUFA and PUFA peroxidation (cluster B). Clusters A and B were both split into two sub-clusters, A1 and A2, and B1 and B2, respectively. However, from the dissimilarity truncation value (dotted horizontal line), only the B1/B2 sub-clustering split was found to be statistically significant.

The more complex aldehydic LOPs present in cluster A, which arise from PUFA and not MUFA peroxidation, include 4-hydroxy-trans-2-alkenals generated from reduction of their corresponding 4-hydroperoxy- derivatives in linoleoylglycerol-rich oils, and 4,5epoxy-trans-2-alkanals such as 4,5-epoxy-trans-2-decenal, which is known to be derived from alka-(trans,trans)-2,4-decadienal [13,22]; the latter dienal arises from fragmentation of linoleate's 9-hydroperoxide [14-18]. Moreover, it is also conceivable that some alka(cis,trans)-2,4-dienals may at least partially be formed from the thermally induced isomerism of their corresponding trans,trans-isomers, and this may explain the co-clustering of these two $\alpha, \beta$-unsaturated aldehydes (sub-cluster A2) [13]. Sub-cluster B1, which contains the cis-2-alkenal aldehyde classification alone, is statistically distinct from sub-cluster B2, which contains a composite of trans-2-alkenals and $n$-alkanals, that are both derived from the peroxidation of both MUFAs and PUFAs, as noted above. However, since all three of these aldehydes are found 'nesting' within a single major cluster, it remains a possibility that cis-2-alkenals may be generated from the thermally mediated isomerism 
of their more predominant trans-isomers. Indeed, plots of its concentration versus LSSFE heating time-point confirmed that this minor aldehyde class was not generated until the later ( $\geq 30 \mathrm{~min}$ ) LSSFE time-points, and only for sunflower and extra-virgin olive oils at 30 min itself (data not shown). This suggests that cis-2-alkenals arise as tertiary LOPs from isomerism of their trans-adducts. For example, in principle cis-2-heptenal may be generated from isomerism of its corresponding trans-isomer, which is a $\beta$-homolysis product of linoleate-12-hydroperoxide [56].

\subsection{Considerations of Possible Evaporative Loss of Culinary Frying Oils during LSSFEs}

With the LSSFEs employed in this study, one concern is the possible evaporative loss of oil triacylglycerols. However, although not directly measured, we noted no significant evaporative losses in oil volume during the course of these experiments. This observation is largely ascribable to the temperature employed for this purpose $\left(180^{\circ} \mathrm{C}\right)$, a value which is significantly lower than the decomposition smoke-points of all the culinary oils investigated (available in Section 2.1). However, the boiling points of tripalmitin, triolein and trilinolein are 315,554 and $816.5^{\circ} \mathrm{C}$, respectively.

One report by Hrncirik and Zeelenberg (2014) [57] revealed that exposure of sunflower and rapeseed oils, and butter and margarine, to shallow frying practices reduced their essential fatty acid (linoleic and linolenic acid) contents by only $\leq 5 \%$, and since these PUFAs are the ones most likely to be lost during such high-temperature processes in view of their peroxidative susceptibilities, in principle we should perhaps expect a maximal evaporative oil loss equivalent to this value per shallow frying session. However, not all fatty acids are lost by evaporation as oxidation products into the gas phase. Indeed, in the above study [57], up to $1.3 \%$ of triacylglycerols were retained within the oil medium as polymeric triacylglycerols (PTGs) following shallow frying.

\subsection{LF Benchtop (60 MHz) NMR Analysis of LOPs in Thermally-Stressed CFOs}

Figure 5 shows the aldehydic (-Cㅌㅇ) function proton regions (9.40-10.05 ppm) of corresponding LF $60 \mathrm{MHz}$ and HF $400 \mathrm{MHz}{ }^{1} \mathrm{H}$ NMR spectra of PUFA-rich soybean oil and SFA-rich pork lard exposed to thermal stressing according to our LSSFEs performed at a temperature of $180{ }^{\circ} \mathrm{C}$ for a 90 min duration. These results demonstrate and confirm the ready applicability of a LF, virtually portable NMR facility for the detection of aldehydic LOPs in reused frying oil and fat media, although both signal resolution and sensitivity are, of course, diminished when using this form of NMR analysis. However, resonances arising from three classes of the most predominant secondary aldehydic LOPs were distinguishable in these $60 \mathrm{MHz}$ spectra, specifically those of trans-2-alkenals, alka-(trans,trans)-2,4dienals and $n$-hexanals. The concentrations of these aldehydes in these thermally stressed soybean oil and lard samples were 7.66 and $5.60 \mathrm{mmol} \mathrm{kg}{ }^{-1}$ trans-2-alkenals; 7.86 and $2.67 \mathrm{mmol} \mathrm{kg}^{-1}$ alka-(trans,trans)-2,4-dienals; and 4.38 and $3.31 \mathrm{mmol} \mathrm{kg}^{-1} \mathrm{n}$-alkanals, respectively. Since alka-(trans,trans)-2,4-dienals arise from the peroxidation of PUFA and not MUFA substrates, much higher levels of this di-unsaturated aldehyde were found in the heated soybean oil sample, as expected. However, ${ }^{1} \mathrm{H}$ NMR signals arising from the -C HO protons of 4,5-epoxy-trans-2-alkenals, 4-hydroxy-/4-hydroperoxy-trans-2-alkenals, and alka-(cis,trans)-2,4-dienals were only detectable in the $400 \mathrm{MHz}$ spectra acquired. 
(a)

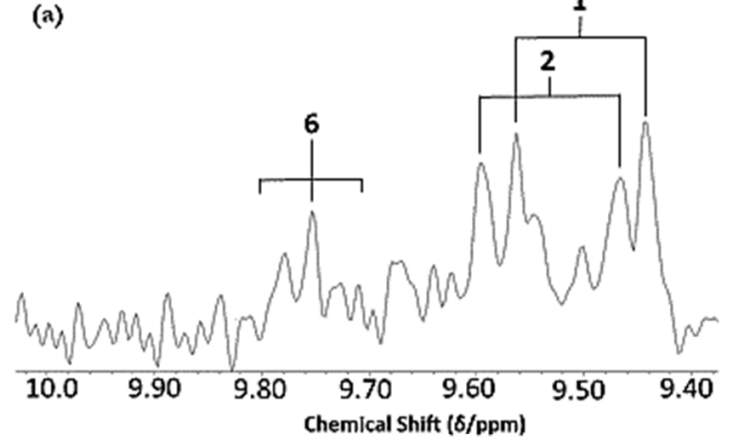

(b)

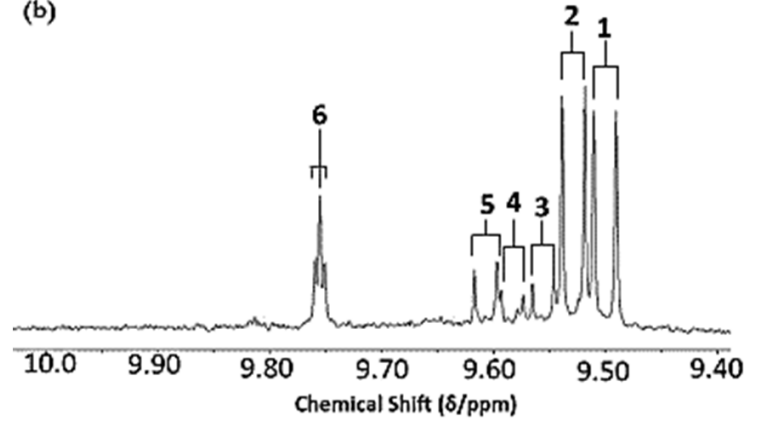

(c)

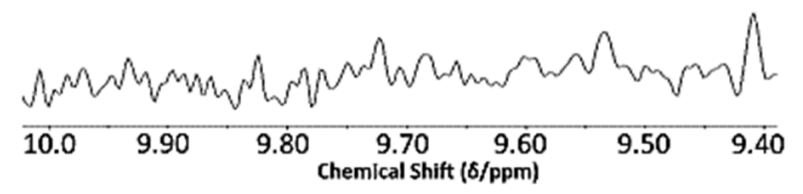

(d)

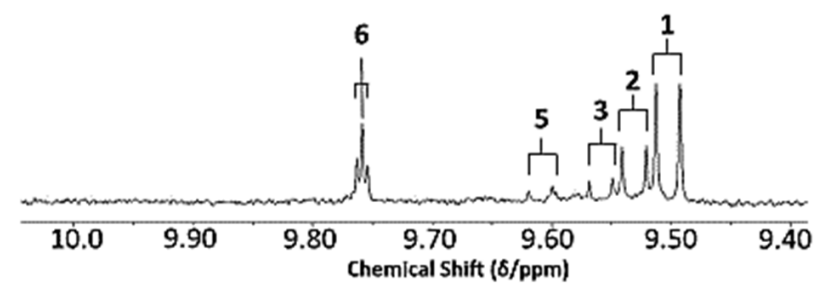

Figure 5. (a,b), Comparative aldehydic-CHO proton regions of the 60 and $400 \mathrm{MHz}{ }^{1} \mathrm{H} \mathrm{NMR}$ spectra, respectively, of soybean oil heated at $180{ }^{\circ} \mathrm{C}$ for 90 min according to LSSFEs. (c,d), as (a,b), respectively, but for a sample of SFA- and MUFA-rich pork lard. The acylglycerol terminal- $\mathrm{CH}_{3}$ function signals in the $400 \mathrm{MHz}$ spectra acquired revealed that the omega-3 FA content of the soybean oil sample decreased from 9.28 to 8.54 molar \% following LSSFE exposure for 90 min. Abbreviations: as Figure 1.

\subsection{Case Study Review: Analytical Advantages Offered by ${ }^{1} \mathrm{H}$ NMR Analysis of LOPs in CFOs}

Results acquired clearly demonstrated that the heating of UK-available seed and further vegetable culinary oil products according to shallow frying practices generates very high levels of extremely toxic aldehydic LOPs, which arise from the thermally stimulated, $\mathrm{O}_{2}$-boosted peroxidation of PUFAs and MUFAs therein; these toxins have been proven to be absorbed from the gut into the systemic circulation following their dietary ingestion [23]. As expected, higher levels of toxic aldehydes are generated in oils rich in the more readily peroxidisable PUFAs, although significant amounts also arise from MUFAs at prolonged heating times (i.e., after one or more significant lag phases). Moreover, only two major classes of aldehydes are produced from the fragmentation of MUFA-derived HPMs (specifically $n$-alkanals and trans-2-alkenals, the former of which are arguably of a lower toxicity than the latter), whereas a much broader pattern of these agents are produced from PUFA-derived CHPD fragmentation, for example 4,5-epoxy-trans-2-alkenals and alka-(trans,trans)-2,4-dienals [14,17]), all of which have the potential to exert a very high level of adverse health effects in humans. Intriguingly, the total unsaturated aldehyde concentration determined in PUFA-rich corn or sunflower oils heated for a period of $90 \mathrm{~min}$ according to our LSSFEs is very close to $50 \mathrm{mmol} \mathrm{kg}^{-1}$, an extremely high level indeed.

This investigation also demonstrates that high-resolution ${ }^{1} \mathrm{H}$ NMR analysis offers many advantages regarding evaluations of the peroxidative sensitivities of commercially available frying oil products when exposed to LSSFEs, or real-life domestic or commercial frying practices. Moreover, as notable from Figure $1 b, c$, it is also of value for determining the stabilities and oxidative resistivities of such oils when exposed to long-term storage episodes at ambient or lower temperatures. This multicomponent analytical technique provides a range of benefits over the established accelerated, heat-dependent Rancimat ${ }^{\circledR}$ method [58], since it provides valuable molecular information on the identities of a variety of LOP classifications (for example, HPMs, CHPDs, isomeric fatty acid epoxides, and up to 10 classes of aldehydic fragmentation products, and all of these within a single ${ }^{1} \mathrm{H}$ NMR 
acquisition which takes ca. 10-15 min). Therefore, this analytical strategy has the ability to support mechanistic investigations of CO UFA peroxidation and/or thermal degradation processes. Hence, NMR analysis enables the rapid, simultaneous, virtually non-invasive, and non-destructive identification and quantification of many major and minor agents that are present in complex, multicomponent CFO samples. Although FTIR analysis, which is now viewed as a 'green' analytical technology [59], is more bench- and sample sourceaccessible than high-resolution NMR analysis, and may be employed for chemometric determinations of cod liver oil product authenticities [60], and their peroxidation status [61], recent technological developments in the operation of non-stationary, near-portable LF benchtop NMR instruments for such culinary oil analysis purposes, as documented here, now provide considerable analytical advantages [62]. Indeed, these facilities have a much improved molecular selectivity, and in at least some cases specificity, over those offered by FTIR techniques.

\subsection{Critical Review of the Reliabilities and Selectivities of Commonly Employed Non-NMR} Methods for LOP Determinations

\subsubsection{Spectrophotometric Conjugated Dienes Assay}

From an analytical food chemistry standpoint, the conjugated dienes (CDs) spectrophotometric assay method employed by researchers, e.g., by Saguy et al. (1996) [63], Sochr et al. (2014) [64] and Opperman et al. (2016) [44], has a very poor selectivity than that afforded by peroxide value (PV) analysis, which is also commonly performed [44]. Likewise, this selectivity is very poor when compared to that of the ${ }^{1} \mathrm{H}$ NMR spectroscopic technologies presented here. This CDs method is based on the absorption of UV light by conjugated double bonds within CHPD molecules (the conversion of PUFAs to such CHPDs involves a rearrangement of $>\mathrm{C}=\mathrm{C}<$ double bonds present in these FAs). However, at the wavelength range employed for this relatively simple test $(230-235 \mathrm{~nm})$, many interfering compounds also absorb in this spectral region, notably carotenoids, tocopherols and phenolic antioxidants, not to mention possible contributions from $\alpha, \beta$ unsaturated aldehydes generated from the fragmentation of CHPDs [65,66], especially the more highly unsaturated ones [67]. Indeed, $\alpha, \beta$-unsaturated aldehydes and ketones have electronic absorption spectra with maxima located at ca. $230\left(\pi \rightarrow \pi^{*}\right.$ absorption, $\varepsilon=$ ca. $1.3 \times 10^{4} \mathrm{M}^{-1} \mathrm{~cm}^{-1}$, where $\varepsilon$ represents molar extinction coefficient), and $310 \mathrm{~nm}$ ( $\mathrm{n} \rightarrow \pi^{*}$ absorption, $\varepsilon=$ ca. $1.5 \times 10^{2} \mathrm{M}^{-1} \mathrm{~cm}^{-1}$ ) [67], and total concentrations of the former aldehyde classifications in repeatedly thermo-oxidised CFOs may attain values as high as $50 \mathrm{mmol} \mathrm{kg}{ }^{-1}$ (approximately equivalent to $15 \mathrm{mmol}$ aldehyde/mol FA). Hence, if the total $\alpha$, $\beta$-unsaturated aldehyde concentration in such an oxidised CFO product is $20 \mathrm{mmol} \mathrm{kg}$, then prior to any dilution it will have absorbance values of ca. 260 and 3 at ca. 230 and $310 \mathrm{~nm}$, respectively. This recommended CD assay system employs a $1 / 600$ dilution with a hexane solvent [63], and hence on consideration of an $\varepsilon$ value of $2.90 \times 10^{4} \mathrm{~mol}^{-1} \mathrm{~cm}^{-1}$ for $\alpha, \beta$-unsaturated aldehydes at $234 \mathrm{~nm}$, the absorbance contribution from these aldehydic interferants alone would be as high as ca. 0.4 at this wavelength, undoubtedly a significant interfering value. However, conjugated triene hydroperoxide species arising from linolenoylglycerol peroxidation absorb strongly at a presumably more specific wavelength of $268 \mathrm{~nm}$ [64].

However, when applied to unheated or unused CFOs, this CD assay system may be suitable, since interferences from aldehydes will be minimal, although not those arising from nutraceuticals such as alpha-tocopherol $(\alpha-\mathrm{TOH})$ and any other phenolic antioxidants present. Indeed, Sochr et al. [64] have reported a strong correlation between CFO hydroperoxide levels and CDs determined by this method. However, it should be noted that HPMs derived from the peroxidation of UFAs are, of course, unresponsive in the CD assay, but are in the classical peroxide value one, and hence the former method's suitability for application to MUFA-rich oils such as olive oil is limited to hydroperoxides derived from PUFAs only.

Therefore, overall such CD assay results are of a limited value, most especially when applied to thermally-stressed CFOs, and hence there is a major call for researchers to 
determine the relative (proportionate) contributions of all interferants, notably those arising from $\alpha, \beta$-unsaturated aldehydes, towards presumed $C D$ absorbance values at $234 \mathrm{~nm}$.

\subsubsection{Spectrophotometric TBARS Test}

The authors of the current paper also continue to express much concern that the spectrophotometrically-based thiobarbituric acid (TBA)-reactive substances (TBARS) test is still neglectfully very frequently employed for determinations of its malondialdehyde (MDA) target analyte, in edible oils and other foodstuffs [68], despite the availability of many reports which clearly demonstrate and confirm the extremely limited value of any results arising therefrom. Indeed, such results are completely unreliable and serve little or no value for the estimation of these species. We are, of course, already aware of the induction of the lipid peroxidation process at standard frying temperatures, and since the TBARS test requires the heating of oil or food extracts with TBA at ca. $96^{\circ} \mathrm{C}$ for periods of $15 \mathrm{~min}$ or more, this temperature is certainly more than sufficient to induce the peroxidation of PUFAs located therein. Hence, in general it appears that all results derivable from this heat-dependent test system represent nothing more than artefactual data. Indeed, our ${ }^{1} \mathrm{H}$ NMR experiments have clearly proven the thermally-induced oxidation of PUFAs in commercial oil formulations to CHPDs and aldehydic LOPs at both Pasteurisation and ambient room temperatures (72 and ca. $25{ }^{\circ} \mathrm{C}$, respectively) [12]. Moreover, further caution should always be employed, since it is known that a series of additional agents present in foods in general and not CFOs, especially reducing and non-reducing sugars, also react with TBA to form chromophores which also absorb strongly at a wavelength of $532 \mathrm{~nm}[69,70]$. Hence, if it was not already producing artefactual results from the thermal stressing of MUFAs and PUFAs, this test's specificity would be less than zero. Attempts to improve the selectivity and specificity of this aberrant test system by the separation of the 2:1 TBA:MDA chromophore from others generated during the heating phase of the assay are of little or no value, however. Indeed, this 'improvement' is, of course, also of negligible value if the MDA is already artefactually generated at the elevated temperatures involved in chromophore development.

If, however, this method involves a prolonged equilibration at ambient temperature, and the 2:1 TBA:MDA chromophore arising $\left(\lambda_{\max } 532 \mathrm{~nm}, \varepsilon=1.55 \times 10^{5} \mathrm{M}^{-1} \mathrm{~cm}^{-1}\right)$ is then allowed to develop slowly, perhaps for $24 \mathrm{~h}$ or more, then this test system does have the potential to monitor aldehydic LOPs in unheated or heated oils, but not exclusively MDA, since a wide range of other aldehydes (including $\alpha, \beta$-unsaturated ones) also react with TBA to generate the same or similar chromophores, or similar interfering adducts.

Notwithstanding, we have previously shown that the careful extraction of watersoluble MDA from thermally-stressed or unheated CFOs into an aqueous medium prior to the above heating stage of the assay serves to overcome this problem [19]. Indeed, visible region electronic absorption spectra acquired from such aqueous extracts demonstrate that the characteristic 2:1 TBA:MDA chromophore absorption bands are clearly distinguished from those of other coloured products present (Figure 6). Moreover, this colourimetric method is also suitable for producing an impacting visual display of aldehyde formation during the heating of UFA-containing CFOs (Figure 7); the intensity of the red colouration formed is, of course, critically dependent on the unsaturation status of the oil tested (with PUFA-rich corn and sunflower oils generating much higher intensities than MUFA-rich olive oil, for example), and the heating exposure period at standard frying temperature (generally, but not exclusively at $180^{\circ} \mathrm{C}$ ). 


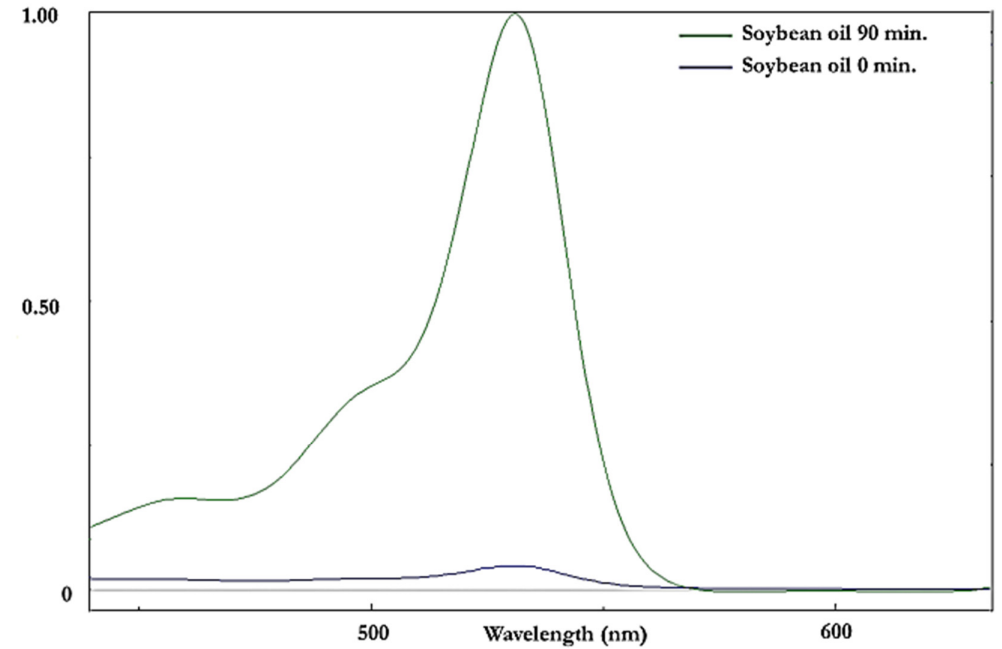

Figure 6. Visible (440-640 nm) region electronic absorption spectra of an aqueous extract of soybean oil derivatised with the TBA reagent both before (mauve spectrum) and after (green spectrum) exposure to a LSSFE at a temperature of $180^{\circ} \mathrm{C}$ for $90 \mathrm{~min}$. The first extraction efficacy of the method employed for interference-free, specific MDA determination [19] was $78 \pm 2 \%$ (mean \pm SEM). The absorbance values at $532 \mathrm{~nm}$ correspond to CFO MDA concentrations of 1.5 and $31 \mu \mathrm{mol} \mathrm{kg}{ }^{-1}$ oil for the 0 and $90 \mathrm{~min}$ LSSFE time-points, respectively.

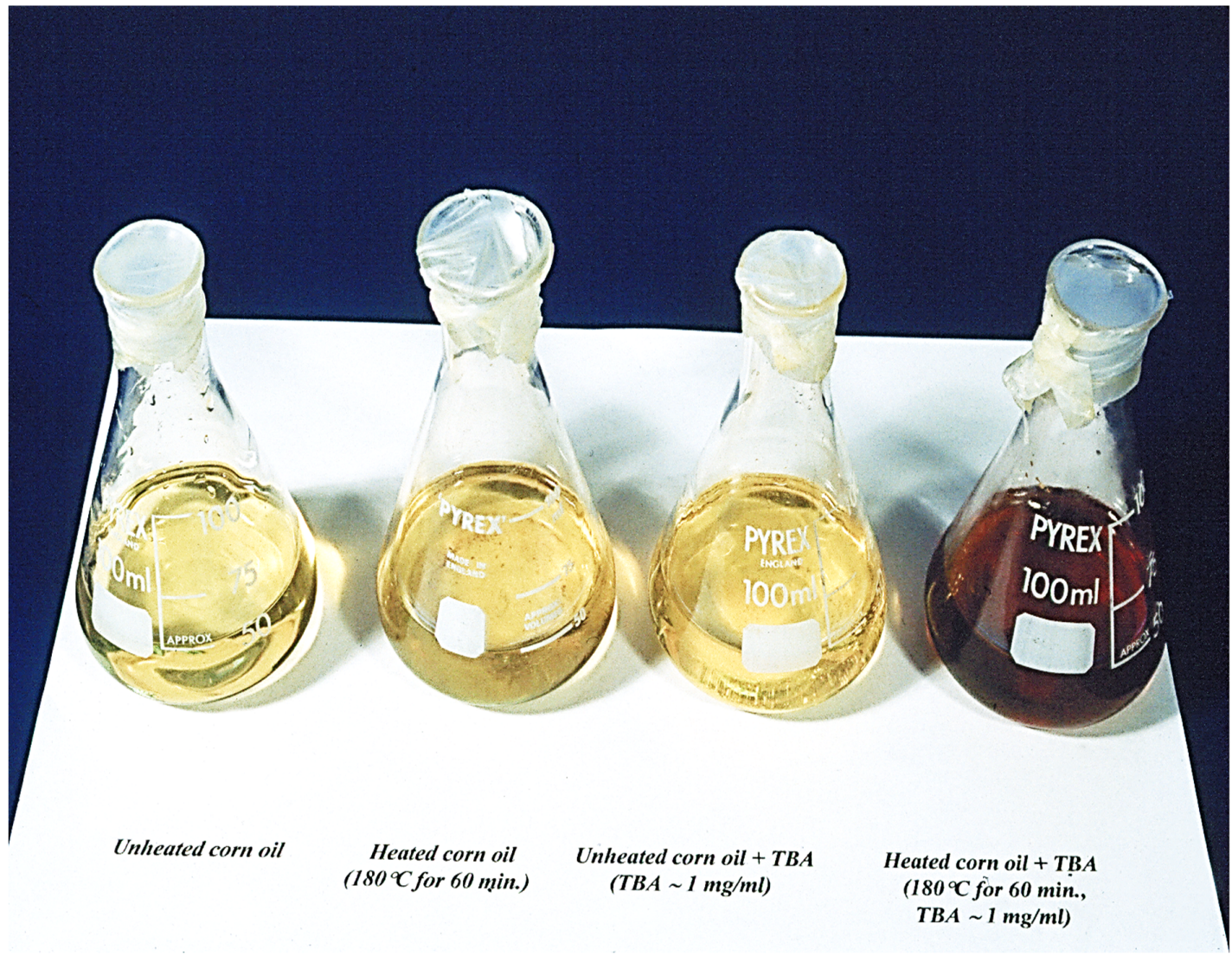

Figure 7. Aldehyde-sensitive visual chromogenic thiobarbituric acid (TBA) test applied to unheated and thermally- stressed corn oil samples. Far left, unheated corn oil; centre left, corn oil heated at $180^{\circ} \mathrm{C}$ for a period of $60 \mathrm{~min}$; centre right, unheated corn oil containing $1 \mathrm{mg} / \mathrm{mL}$ TBA reagent; far right, corn oil heated at $180^{\circ} \mathrm{C}$ for a 60 min duration, but following addition of TBA (final added level $1.0 \mathrm{mg} / \mathrm{mL}$ ). Photograph taken at Prof. Grootveld's laboratory based at St. Bartholomew's and the Royal London Hospital School of Medicine and Dentistry, circa 1995. Reproduced from Ref. [11] with permission. 


\subsection{Considerations of General Health Risks Posed by Human Exposure to Aldehydic LOPs \\ 3.7.1. Overview of Health Risks}

In view of these serious public health concerns presented by aldehydic toxins present in thermally-stressed CFOs, we refer readers to a series of hazard pictograms and corresponding descriptions that, by law, accompany the purchase of cytotoxic and genotoxic aldehyde products as laboratory reagents, etc.; these relate to the labelling and packaging of these chemicals, and serve as austere warnings to laboratory workers (together with others involved in their handling), and also as a means of controlling risks to such personnel [71]. Such health and safety warning labels for typical reference saturated and $\alpha, \beta$-unsaturated aldehyde products available for purchase from chemical companies are available at $\mathrm{Haz}$ ard symbols and hazard pictograms-Chemical classification-HSE. http:/ /www.hse.gov.uk (accessed on 11 March 2020) [71]. Labels applied to aldehyde chemical products generally involve an absolute minimum of one, and more often several or more of these labels, which are defined as (1) acute toxicity-oral, dermal, inhalation (categories 1, 2 and 3); (2) respiratory sensitization (category 1), germ cell mutagenicity (categories 1A,1B and 2), carcinogenicity (categories 1A,1B and 2), reproductive toxicity (categories 1A,1B and 2), specific target organ toxicity-single and repeated exposures (both categories 1 and 2), and aspiration hazard; (3) acute toxicity (category 4), skin and eye irritation, skin sensitization, specific target organ toxicity, and respiratory tract irritation; (4) hazardous to the aquatic environment-acute hazard (category 1), and chronic hazard (categories 1 and 2); (5) corrosive to metals (category 1), skin corrosion (categories $1 \mathrm{~A}, 1 \mathrm{~B}$ and $1 \mathrm{C}$ ), and serious eye damage (category 1); and finally (6) flammability and self-reactivity (the former consisting of a wide range of categories). However, the nature and number of such hazard pictograms applied are aldehyde class- and product-dependent.

On consideration of these grave alerts, the authors believe that this information is more than sufficient to justify the toxicological and associated adverse health concerns addressed here, and also to warrant or stimulate further research work in the clinical nutrition, public health and population toxicology arenas.

For acetaldehyde, one of the structurally simplest $n$-alkanals, and which is arguably significantly less toxic than $\alpha, \beta$-unsaturated aldehydes, these hazard warnings comprise: class 4 acute toxicity substance (ingestion); class 2 severe injury/eye irritation substance; class 2 reproductive cell mutagenicity substance; class 2 carcinogen; and class 2 specific target organ systematic toxicity (repeated exposure). Furthermore, it is also a class 1 flammable liquid. For acrolein, however, the labels featured are those described under descriptors (2), (3) and (4) above.

3.7.2. Estimated Daily Dietary Intakes of Aldehydic LOPs: Accordance with Governmental Regulatory Limits?

Although the lipid content of fried products is highly dependent on the type of food, frying episode class (e.g., deep- versus shallow-frying), frying oil used, frying time duration and frying temperature, these values generally range from $6-38 \%(w / w)$ [72-74], and as expected, the FA compositions of CFOs substantially alters that of foods fried therein, as would be expected with significant levels of CFO infiltration, for example those observed for potato chips [72,75] and fish fillets [76]. Hence, frying oil acylglycerol-normalised (proportionate) concentrations of LOPs will also be expected to migrate into foods fried in such media, and in 2015 Csallany et al. [77] reported that peroxidised linoleoylglycerolderived HNE was readily detectable in French fry samples collected from $n=6$ fast-food restaurants at concentrations of $8-32 \mu \mathrm{g} / 100 \mathrm{~g}$ portions (equivalent to $0.51-2.05 \mu \mathrm{mol} \mathrm{kg}{ }^{-1}$, and $0.9-4.9 \mu \mathrm{g} / \mathrm{g}$ of extracted lipid). Therefore, for a very large (adult) serving of European fried potato chips of mass $400 \mathrm{~g}$, this represents between 32 and $128 \mu \mathrm{g}$ of HNE. However, an important consideration is that this aldehyde, which is PUFA- and not MUFA-derived, is generally detectable in thermally-stressed PUFA-containing oils at much lower levels than those of similarly health-threatening trans-2-alkenals and alka-(trans,trans)-2,4-dienals. From our laboratory, typical estimates of total 4-hydroxy-trans-2-alkenals expressed as a 
percentage of the total $\alpha, \beta$-unsaturated aldehyde content remaining in oils when heated at $180{ }^{\circ} \mathrm{C}$ for a 90 min duration are only ca. $<2$ and $7 \%$ for extra-virgin olive and sunflower oils, respectively (data not shown).

Mean concentrations of total trans-2-alkenals, alka-(trans,trans)-2,4-dienals and $n$-alkanals in potato chip samples purchased from fast-food restaurants, which were found to be 121, 157 and $126 \mu \mathrm{mol} \mathrm{kg}{ }^{-1}$, respectively [13], are certainly substantially greater than those of 4-HNE. Moreover, these levels are also markedly higher than trace levels of acrylamide found in French fry and potato snack foods, which are predominantly lower than the regulatory limit value set by the European Commission (EC) for ready-to-eat fries, i.e., $0.60 \mathrm{ppm}$ (equivalent to only $8.4 \mu \mathrm{mol} / \mathrm{kg}$ ) [78], and which rarely exceed $1 \mathrm{ppm}$ (equivalent to $\left.14.1 \mu \mathrm{mol} \mathrm{kg}{ }^{-1}\right)$. Acrylamide is known to exert toxicological actions on the nervous system and fertility, and is also potentially carcinogenic [79]. A further important point is that acrolein may serve as a significant acrylamide source when asparagine-rich foods are deep-fried in PUFA-rich oils [80].

In this context, it is therefore important to note that recently the Australian Government Department of Health specified that the acceptable daily intake (ADI) value for acrolein (which can be considered to be the lowest homologue $\alpha, \beta$-unsaturated aldehyde, of a similar toxicity to other $\alpha, \beta$-unsaturated aldehydic LOPs), i.e., that which is considered to be a level of intake of this molecule that can be ingested daily over an entire lifetime without any appreciable risk to health, to be only $0.5 \mu \mathrm{g}$ per $\mathrm{kg}$ of body weight (BW), i.e., a total of only $35 \mu \mathrm{g}$ for an assumed (average) human BW of $70 \mathrm{~kg}$ [81]. The corresponding value set by the World Health Organisation (WHO) [82] is $7.5 \mu \mathrm{g}$ per $\mathrm{kg}$ BW, i.e., $525 \mu \mathrm{g}$ for an average $\mathrm{BW}$ of $70 \mathrm{~kg}(\mathrm{WHO}, 2002)$. This consideration alone is a very worrying prospect, not least because this value represents the human intake of only one of many toxic/carcinogenic aldehydes available in fried meals. In [13], we estimated the mean \pm SEM acrolein molecular mass-normalised contents of the most predominant $n$-alkanals, trans-2-alkenals and alka-(trans,trans)-2,4-dienals contained within a typical $154 \mathrm{~g}$ 'large' portion of European fried potato chips obtained from fast-food restaurants (i.e., those arising from oxidation of the highest content PUFA in a PUFA-predominant CFO such as corn or sunflower oils, specifically linoleic acid as linoleoylglycerols), which were found to be as high as $1.5 \pm 0.2$ $(2.6 \pm 0.4), 1.1 \pm 0.2(2.5 \pm 0.4)$ and $1.1 \pm 0.2(2.9 \pm 0.4) \mathrm{mg}$, respectively (actual estimated values are in brackets) for $n$-hexenal, trans-2-octenal and deca-(trans,trans)-2,4-dienal, contents substantially greater or greater than the recommended ADI values documented by the AGDH and the WHO, respectively [83]. It should also be noted that these estimates are for only a single serving of this very commonly consumed fried food source. However, as noted above, for some Western world populations, these estimates remain conservative, since 300-400 g servings of potato chips are indeed quite common. Astoundingly, these estimated $154 \mathrm{~g}$ potato chip serving aldehyde levels are quite similar to those ingested during the smoking of a daily 25 tobacco cigarette allocation, for example crotonaldehyde (1.8-5.7 $\mathrm{mg}$ ) and $n$-hexanal (2.5-9.5 $\mathrm{mg})$, respectively [84].

The National Institute of Occupational Safety and Health's (NIOSH's) Immediately Dangerous to Life or Health (IDLH) Limit is an occupational health index, i.e., the NIOSH's recommended exposure limit to ensure that a worker may escape from such an exposure situation that is likely to cause death or immediate or delayed permanent adverse health effects, or prevent escape from the environment. In 1994, the NIOSH [85] revised its IDLH

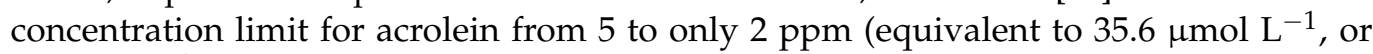
$4.58 \mathrm{mg} / \mathrm{m}^{3}$ for its gaseous, inhalable form (b.pt $\left.53^{\circ} \mathrm{C}\right)$ ). Indeed, they quote 'Human data: It has been reported that $5.5 \mathrm{ppm}$ [of acrolein] results in intense irritation and marked lacrimation, after $60 \mathrm{~s}$ [86]. Exposures to $1.8 \mathrm{ppm}$ result in slight eye irritation after $1 \mathrm{~min}$ and profuse lacrimation after $4 \mathrm{~min}$ [87]. In volunteers exposed for $5 \mathrm{~min}$, concentrations of 2 to 2.3 ppm produced severe irritation [88]. A 10 min exposure at 8 ppm and a 5-min exposure at $1.2 \mathrm{ppm}$ elicited extreme irritation described as "only just tolerable"' [89]. This revised IDLH value is based on the above acute inhalation toxicity data available for humans, so those exposed to or potentially exposed to cooking/frying oil fumes containing 
acrolein and similarly toxic $\alpha, \beta$-unsaturated aldehydes should exert a high level of future caution regarding this potentially very serious health threat.

Corresponding IDLH values for acetaldehyde, formaldehyde and furfural confirmed or revised in 1994 are 2000, 20 and 100 ppm [83]. Intriguingly, that for hydrogen cyanide $(\mathrm{HCN})$ is $50 \mathrm{ppm}$, i.e., as much as 25 -fold greater than that for acrolein [85].

The NIOSH recommendation for the permissible exposure to acrolein in our air supply is $0.10 \mathrm{ppm}\left(0.25 \mathrm{mg} / \mathrm{m}^{3}\right)$ for a $8.0 \mathrm{~h}$. duration time-weighted average (TWA) concentration; TWAs represent threshold limit values (TLVs) based on a $8 \mathrm{~h}$. workday, and a $40 \mathrm{~h}$. work week. The Australian, Mexican, Israeli and Canadian Provincial (i.e., Alberta, British Columbia, Ontario and Quebec) TWA and short-term exposure limit (STEL) indices are the same as the above NIOSH limit, whereas Israel has an action limit of only $0.05 \mathrm{ppm}$; STEL values are TLVs based on a $15 \mathrm{~min}$ average.

\subsubsection{Carcinogenicities of Selected $\alpha, \beta$-Unsaturated Aldehydes}

Currently, there is powerful evidence available that acrolein displays manifold key characteristics of carcinogens, which has been primarily acquired from human primary cell investigations, along with a range of experimental systems. This evidence has been strongly supported by human studies focused on structural molecular and bioanalytical explorations of acrolein's DNA adducts. Inhaled acrolein has been demonstrated to be carcinogenic in two species of rodent, it induces malignant lymphoma in female B6D2F1/Crlj mice [90,91], and has been shown to enhance the incidence of a composite of rare nasal cavity rhabdomyoma and squamous cell carcinoma in female F344/DuCrlCrlj rats [89]. Acrolein readily reacts with DNA bases to form covalent DNA adducts, including cyclic $\alpha$ - and $\gamma$-hydroxy-1, $\mathrm{N}^{2}$-propanodeoxyguanosine derivatives (the former is detectable in a variety of human biosamples, for example saliva, lung, brain liver, and urothelial mucosa) $[92,93])$. Upregulated concentrations of such DNA adducts have been observed in tobacco-smoking humans [92,94], and also those with chronic inflammatory conditions.

Similarly, there is "strong" evidence that crotonaldehyde exhibits multiple key characteristics of carcinogens, and again this arises from studies conducted in human primary cells and a wide range of experimental systems, which are supported by those focused on DNA adduct formation and proliferation in humans. Notwithstanding, to date there remains only "limited" evidence for its carcinogenicity in experimental animals. Nevertheless, one notable study from 1986 [95] reported that it increased the incidence of hepatocellular adenoma and carcinoma combined in male F344 rats when administered in drinking water. Moreover, this $\alpha, \beta$-unsaturated aldehyde is genotoxic [96], and exerts clastogenic properties in human primary cells and human cell lines [96].

In November 2020, a Working Group representing the International Agency for Research on Cancer (IARC) finalized their evaluations of the carcinogenic potentials of acrolein and crotonaldehyde, along with arecoline (the primary active ingredient of the areca nut). Acrolein was classified as "probably carcinogenic to humans" (Group 2A) in view of "sufficient" evidence of carcinogenicity in experimental animals and "strong" mechanistic evidence. However, crotonaldehyde and arecoline were classified as "possibly carcinogenic to humans" (Group 2B) on the grounds of "strong" mechanistic evidence [97]. The deleterious toxicological effects of acrolein are further reviewed in detail in [98].

3.7.4. Toxicological Significance of Dietary LOPs in CLOs and CLO-Loaded Fried Foods, and Its Frying Process Type-Dependence

Although it appears that a significant level of the total aldehydes generated from PUFA and, to a much lesser extent, MUFA peroxidation processes in both thermally- stressed culinary oils, and via transference to foods fried therein, are depleted by their potential participation in Maillard and/or Michael addition chemical reactions with food matrix proteins and amino acids [13], these secondary LOPs clearly remain a major toxicological concern. Indeed, the above examples provided by $[77,83]$ report only the levels of 'free' aldehydic LOPs available for human consumption, and hence the total amount uptaken by the fried potato chip samples analyzed in these investigations may indeed be significantly, 
or even substantially, greater. Moreover, such Maillard or Michael addition products could also serve as latent sources of bioavailable and bioactive aldehydes [99], a process which may promote or even prolong their deleterious toxic actions in vivo following ingestion. Similarly, chemical reactions of aldehydes with alcohols, polyols or even carbohydrates may yield hemiacetal and subsequently acetal species in fried foods; however, such reactions are readily reversible.

Our previous ${ }^{1} \mathrm{H}$ NMR-based studies have also demonstrated that shallow frying practices give rise to much greater levels of LOPs than those observed during deep frying episodes performed under the same experimental conditions and temperature. This observation reflects the influence of the surface area of the frying medium, and hence its exposure to atmospheric $\mathrm{O}_{2}$, and also the subsequent dilution of LOPs generated into the bulk frying medium [12]. However, in a toxicological context, the lower levels of aldehydes found in deep-fried culinary oils may, at least in part, be compromised by the greater extent of oil absorption or exchange by the fried food matrix under these frying conditions.

Intriguingly, concentrations of aldehydes arising from the thermal stressing of commercially available, PUFA- or MUFA-rich culinary oils is a value representing only that remaining therein $[12,13,15,19]$. Certainly, a large number of the aldehydes generated are volatilised at standard frying temperatures, and this may also present serious health hazards in view of their inhalation by humans, especially those working in fast-food retail outlets or restaurants with insufficient or inadequate ventilation precautions, or those performing classical Chinese-style wok cooking episodes domestically. This is especially the case for aldehydes arising from the peroxidation of linoleoyl- and linolenoyglycerols (particularly deca-trans,trans-2,4-dienal and acrolein, respectively), since the great majority of aldehydic secondary LOPs generated therefrom have boiling-points (b.pts) $<180{ }^{\circ} \mathrm{C}[49]$, at least some of them substantially so.

In view of the above considerations, the 'safety' criteria outlined by many researchers', e.g., those provided in [44] are therefore an unrealistic representation, i.e., one which furnishes an incomplete message to scientists, readers and consumers regarding the consumption of fried foods laden with the oils in which they have been heated according to either shallow- or deep-frying processes, and therefore also the proportionate, frying oil FA unsaturation status-dependent concentrations of LOPs contained therein.

Notwithstanding, unless they have been exposed to such frying practices, or alternatively stored for prolonged periods of time at ambient temperature and/or exposed to light, we agree that PUFA-rich vegetable seed oils offer little or no threats to human health; indeed, they contain essential FAs such as linoleic and $\alpha$-linolenic acids. However, since some researchers focused on the positive health benefits offered by PUFA-rich CFOs appear not to have considered the thermally induced generation of LOPs during frying practices, such 'safety' arguments on this point are moot, and poorly representative of 'real-life' scenarios. As an example, Ref. [44] shows the lipid hydroperoxide (i.e., HPM plus CHPD) levels of these oils before they have been exposed to either shallow- or deepfrying practices: if that is the concentration of these agents before exposure to such high temperature stresses, how much will be present thereafter, let alone those of cytotoxic and genotoxic aldehydes generated from their fragmentation? This is therefore a major scientific as well as public health concern.

The authors of [44] correctly state that ' ... animal fat is also prone to degradation during cooking since it does not consist of saturated fat only, but is rather a combination of SFA, MUFA and PUFA, in different ratios'; however, they do not indicate that such fats contain much lower PUFA contents than those of commonly employed seed or other vegetable oils (only ca. $5 \%(w / w)$ for pork or beef tallows), and are therefore much less susceptible to oxidative damage during standard frying or cooking practices. These researchers, together with many others, also attempt to rationalize and emphasize the critical importance of omega- 6 to omega-3 FA ratios in the oils tested as a 'health index' parameter, with 'desirable' values of 2.0-5.1 quoted [1]. However, the most predominant omega-3 FA in the oils explored, $\alpha$-linolenic acid, which has three $-\mathrm{CH}=\mathrm{CH}$ - and two bis-allylic- $\mathrm{CH}_{2}$ - functions, is 
substantially susceptible to oxidation during standard frying/cooking episodes (i.e., more than twice so that of linoleic acid, which has only two such carbon-carbon double bonds and one bis-allylic- $\mathrm{CH}_{2}$ - group [14]), and therefore this ratio is virtually meaningless if humans are consuming a lot less omega-3 FAs than expected when ingesting fried foods loaded with such thermally stressed culinary oils (and perhaps also significantly lower amounts of linoleic acid if the oils have been thermally stressed for extended periods [13]).

3.7.5. Potential Suppression of LOP Generation during High-Temperature Frying Practices: Do Dietary Antioxidants Work?

A further important factor is that the concentrations of natural or oil-supplemented lipid-soluble, chain-breaking dietary antioxidants such as $\alpha-\mathrm{TOH}$ (vitamin E), butylated hydroxytoluene (BHT) and DTBHQ, molecules, which are known to block the lipid peroxidation process leading to the generation of toxic aldehydes, unfortunately appear to be only poorly effective at suppressing such toxic LOP production during standard frying practices $[12,23]$. This is primarily ascribable to the inability of such low antioxidant concentrations to combat the aggressive, autocatalytic oxidative assault upon peroxidatively susceptible PUFAs induced at such high temperatures. Indeed, reports available confirm substantial losses of $\alpha-\mathrm{TOH}$ and total phenolic antioxidants in virgin olive and sunflower oils when heated according to standard frying practices [100-102]. Additionally, volatilisation of such antioxidants, together with their thermal instabilities in at least some cases, undoubtedly also contribute to such losses $[23,103]$. Therefore, the contention of some that such antioxidants protect against the peroxidation of PUFAs present in such oils may generally be only relevant to those that are subjected to prolonged periods of storage at ambient or lowered temperatures, and clearly not to the same CFO products exposed to standard or indeed more rigorous frying episodes.

Notwithstanding, it certainly does appear that some non-classical lipid-soluble antioxidants (i.e., non-phenolics), either pre-added or available naturally, may indeed offer a significant level of protection of CFO and marine oil PUFAs against such thermally-induced oxidative stressing episodes [104]. Such antioxidants, for example selected biogenic amines (BAs) present naturally in fermented food products, act via a different mechanism to that of classical phenolic agents, and for linoleoylglycerols, this involves the direct neutralisation of primary carbon-centred pentadienyl source radicals required for triggering the lipid peroxidation process. Moreover, BAs such as 2-phenylethylamine and tyramine have b.pts of 195 and $206^{\circ} \mathrm{C}$, respectively, and hence their longevities in high-temperature $\left(180^{\circ} \mathrm{C}\right)$ frying media may be prolonged over those of other phenolic antioxidants (in view of its phenolic-OH substituent, tyramine may also function via the classical lipid hydroperoxide$\mathrm{OOH}$ function-consuming route). Selected BAs may also have more favourable resistivities against thermal degradation than those of synthetic phenolic antioxidants such as BHT [23].

\subsubsection{Comparative Evaluations of Health Risks Presented by Dietary LOPs and Trans-FAs}

Contributions by researchers concerning the provision of information on trans-fatty acid (TFA) levels in edible oils are of much value, especially since evidence relating TFA intake to the development and progression of cardiovascular diseases remains widespread, and their potential health risks in this context are currently considered to be more substantial than those presented by SFAs [105]. However, in view of these estimates, it is of much importance to note that, on a mole-for-mole basis, aldehydes arising from UFA oxidation are markedly more toxic than TFAs, although the estimated dietary intake of the latter is clearly much greater, or at least it will be in societies and countries which have not or not yet set regulatory legislation to remove them from their food supplies.

Hence, without any rigorous control for the confounding deleterious health effects associated with the intake of aldehydes and further toxic LOPs, along with the concentrations of each of these toxins available in the dietary patterns considered, then such public health investigations targeting TFAs as 'malefactor molecules', particularly those focused on their ability to promote CHDs, may be compromised. Moreover, in principle, TFAs may themselves be susceptible to peroxidative damage, followed by sequential fragmentation of 
any hydroperoxides arising therefrom to corresponding toxic secondary LOPs, including, but not exclusively, aldehydes.

Despite some major conjecture in the literature available, there remains the possibility that the heating of CFOs according to frying practices may convert natural cis-configuration FAs to their corresponding TFA derivatives. One investigation reported a small increase in corn oil TFA levels following its exposure to stir-frying sessions [106].

A recent WHO report [107] specifies that to date, 58 countries have introduced laws focused on the elimination of industrially generated TFAs from the global food supply, and this will protect an estimated 3.2 billion individuals against the harmful effects of these agents prior to the end of 2021. However, more than 100 countries are still required to act on this health-favourable incentive; moreover, $11 / 15$ countries with the highest incidences of CHDs, apparently arising from TFA ingestion, are yet to take such actions.

If that's the case for TFAs, what actions and regulatory legislation will be implemented in order to circumvent the deleterious generation and human ingestion of much more highly toxic dietary LOPs?

\section{Conclusions}

In conclusion, the heating of PUFAs and, to a much lesser extent, MUFAs present in EU-available seed and other CFO products according to high temperature standard frying practices, which predominantly represents the prime purpose for which they are used by consumers, substantially promotes their peroxidation, a process giving rise to the generation of very high levels of cytotoxic and genotoxic aldehydes. The ${ }^{1} \mathrm{H}$ NMR analysis of these samples, which was also performed for one of the first times on a low-field $(60 \mathrm{MHz})$ benchtop spectrometer, confirmed the thermally promoted, time-dependent production of a range of aldehydic LOPs in all oils investigated, the highest levels being observed in PUFA-rich sunflower or corn oils. Lower quantities of these LOPs were formed in monounsaturated fatty acid (MUFA)-rich canola and extra-virgin olive oils, and their generation was preceded by one or more significant lag phases. As expected, only low levels of selected aldehydes were formed in SFA-laden coconut oil during these laboratory-simulated shallow frying episodes.

Advantages offered by the ${ }^{1} \mathrm{H}$ NMR method employed for multicomponent LOP analysis were comparatively reviewed against those of two routinely employed spectrophotometric methods available (the CDs and TBARS spectrophotometric methods). Indeed, such analytical NMR technologies clearly offer major advantages over these alternative methods, most especially in the context of their ability to identify and determine a wide range of these toxins simultaneously, together with their UFA lipidic precursors and minor species (sample preparation is rapid and facile). It is therefore concluded that at least some of the current spectrophotometric/colourimetric laboratory methodologies available for determining and monitoring toxic LOPs in unused or used CFOs are unspecific and poorly quantitative in view of the adverse artefactual generation of such oxidation products during sample preparation phases (e.g., the heating stage of the TBARS assay), or the undesirable incorporation of positively responding interferants in simpler spectrophotometric assays such as those involving oil CD determinations.

This study also revealed the potential applications of $\mathrm{LF}(60 \mathrm{MHz})$, near-portable NMR spectrometers for evaluating the quality and acylglycerol FA status of neat, used and unused CFOs, and the determination of aldehydic LOPs in such frying media. Indeed, such an approach may be suitable for 'on-site' use by edible oil manufacturers, both large and small, together with fast-food restaurants. One further, albeit novel, application is the direct, multicomponent analysis of such CFOs frequently used by street vendors in African nations and elsewhere, and in principle such analyses could be delivered and provided at the point-of-contact by suitably equipped, road-based mobile laboratory transport units.

The gastrointestinal tract is continually exposed to such toxic aldehydes, and subsequent to digestion they are absorbed into the lymphatic system or directly into the systemic circulation [23]. A further important consideration is that the wealth of previous 
investigations focused on the possible beneficial health effects of dietary PUFAs should be dissected and revisited, particularly those featuring feeding trials with humans or animals or, alternatively, associated epidemiological and cohort meta-analysis ones. On reflection, it certainly appears that many of these previously conducted studies may indeed have been flawed, since researchers involved have either ignored or neglected the confounding adverse health effects associated with LOPs (which undoubtedly were present in the oils or diets involved), together with the molecular nature and concentrations of such agents therein. Hence, data acquired here provide strong evidence that previous reports focused on the negligible or limited health risk status of such PUFA-rich CFOs when employed for frying purposes, and that foods fried therein are safe for human consumption, may be erroneous and inaccurate since they fail to consider the multitude of LOPs detectable therein, along with their very broad spectrum of adverse health effects, notably those contributing towards the development and progression of non-communicable chronic diseases such as cardiovascular and neurodegenerative diseases, and cancer.

Future clinical feeding trial or epidemiological investigations focused on explorations of the relationships between the incidence and/or severity of selected chronic human diseases such as CHD, and the frequency and level of dietary LOP ingestion, may indeed serve to delineate the nature of such associations.

Author Contributions: Conceptualization, M.G. (Martin Grootveld); Methodology, Author Contributions: B.C.P., S.M., M.G. (Miles Gibson), K.W., A.A., M.W. and M.E.; Software, M.G. (Martin Grootveld), B.C.P., S.M., M.G. (Miles Gibson), M.E.; Validation, M.G. (Miles Gibson), M.G. (Martin Grootveld), B.C.P. and M.E.; Formal Analysis, B.C.P., S.M., M.G. (Miles Gibson), K.W., A.A., M.G. (Martin Grootveld) and M.E.; Investigation, B.C.P., S.M., M.G. (Miles Gibson), K.W., M.W., A.A., K.L.G., M.G. (Martin Grootveld) and M.E.; Resources, M.G. (Martin Grootveld); Data Curation, M.G. (Miles Gibson), B.C.P. and M.G. (Martin Grootveld); Writing-Original Draft Preparation, M.G. (Martin Grootveld) and M.W.; Writing and Review and Editing, M.G. (Martin Grootveld), M.W., M.E., K.L.G., B.C.P.; Visualization, M.E., A.A., S.M. and M.G. (Martin Grootveld); Supervision, M.G. (Martin Grootveld); Project Administration, M.G. (Martin Grootveld). All authors have read and agreed to the published version of the manuscript.

Funding: This research was part-funded by a De Montfort University (DMU) HEIF award (grant number HE.0044.01.04.06).

Institutional Review Board Statement: Not applicable.

Informed Consent Statement: Not applicable.

Data Availability Statement: Data supporting the reported results of this study may be obtained by correspondence with the correspondence author (mgrootveld@dmu.ac.uk).

Acknowledgments: The authors are very grateful to DMU, Leicester, UK for the provision of highresolution NMR facilities, and also for the provision of HEIF funding to support this project (grant number HE.0044.01.04.06). We also thank the International Trade Centre, United Nations-WTO (Geneva, Switzerland) for non-financial support.

Conflicts of Interest: None of the other authors declare any conflict of interest. The sponsoring body had no role in the design, execution, interpretation, or writing of the study.

\section{References}

1. Simopoulos, A.P. The importance of the omega-6/omega-3 fatty acid ratio in cardiovascular disease and other chronic diseases. Exp. Biol. Med. 2008, 233, 674-688. [CrossRef]

2. FAO/WHO. Fats and Fatty Acids in Human Nutrition. Report of an Expert Consultation; FAO/WHO: Geneva, Switzerland, 2010.

3. Farvid, M.S.; Ding, M.; Pan, A.; Sun, Q.; Chiuve, S.E.; Steffen, L.M.; Willett, W.C.; Hu, F.B. Dietary linoleic acid and risk of coronary heart disease: A systematic review and meta-analysis of prospective cohort studies. Circulation 2014, 130, 1568-1578. [CrossRef] [PubMed]

4. Calder, P.C. Functional roles of fatty acids and their effects on human health. J. Parenter. Enter. Nutr. 2015, 39 (Suppl. 1), 18S-32S. [CrossRef] [PubMed]

5. Bengmark, S. Choose right carbohydrates and right fats (RCRF)—Keys to optimal health. Hepatobil. Surg. Nutr. $2017,6,429-433$. [CrossRef] [PubMed] 
6. Poudyal, H.; Brown, L. Should the pharmacological actions of dietary fatty acids in cardiometabolic disorders be classified based on biological or chemical function? Prog. Lipid Res. 2015, 59, 172-200. [CrossRef] [PubMed]

7. Orsavova, J.; Misurcova, L.; Jarmila, A.V.; Vicha, R.; MLMLcek, J. Fatty acids composition of vegetable oils and its contribution to dietary energy intake and dependence of cardiovascular mortality on dietary intake of fatty acids. Int. J. Mol. Sci. 2015, 16, 12871-12890. [CrossRef] [PubMed]

8. U.S. Department of Health and Human Services and U.S. Department of Agriculture. 2015-2020 Dietary Guidelines for Americans, 8th ed.; HHS Publication \#: HHS-ODPHP-2015-2020-01-DGA-A USDA Publication \#: Home and Garden Bulletin No. $232 ; 2015$. Available online: http:/ / health.gov/dietaryguidelines/2015/guidelines (accessed on 4 June 2019).

9. Estévez, M.; Li, Z.; Soladoye, O.P.; Van-Hecke, T. Health Risks of Food Oxidation. Adv. Food Nutr. Res. 2017, 82, 45-81. [CrossRef]

10. Grootveld, M.; Silwood, C.J.L.; Addis, P.; Claxson, A.W.D.; Bonet Serra, B.; Viana, M. Health effects of oxidised heated oils. Foodserv. Res. Int. 2001, 13, 39-53. [CrossRef]

11. Grootveld, M.; Ruiz-Rodado, V.; Silwood, C.J.L. Detection, monitoring and deleterious health effects of lipid oxidation products generated in culinary oils during thermal stressing episodes. Inform AOCS 2014, 25, 614-624.

12. Haywood, R.M.; Claxson, A.W.D.; Hawkes, G.E.; Richardson, D.P.; Naughton, D.P.; Coumbarides, G.; Hawkes, J.; Lynch, E.J.; Grootveld, M.C. Detection of aldehydes and their conjugated hydroperoxydiene precursors in thermally-stressed culinary oils and fats: Investigations using high resolution proton NMR spectroscopy. Free Radic. Res. 1995, 22, 441-482. [CrossRef]

13. Moumtaz, S.; Percival, B.C.; Parmar, D.; Grootveld, K.L.; Jansson, P.; Grootveld, M. Toxic aldehyde generation in and food uptake from culinary oils during frying practices: Peroxidative resistance of a monounsaturate-rich algae oil. Sci. Rep. 2019, 9, 4125. [CrossRef]

14. Frankel, E.N. Volatile lipid oxidation-products. Prog. Lipid Res. 1983, 22, 1-33. [CrossRef]

15. Choe, E.; Min, D.B. Mechanisms and factors for edible oil oxidation. Compr. Rev. Food Sci. Food Saf. 2006, 5, 169-186. [CrossRef]

16. Dobarganes, M.C.; Perez-Camino, M.C. Fatty acid composition: A useful tool for the determination of alteration level in heated fats. Rev. Fr. Corps Gras 1988, 35, 67-70.

17. Frankel, E.N.; Selke, E.; Nef, W.E.; Miyashita, K. Autoxidation of polyunsaturated triacylglycerols. IV. Volatile decomposition products from triacylglycerols containing linoleate and linolenate. Lipids 1992, 27, 442-446. [CrossRef] [PubMed]

18. Neff, W.E.; Byrdwell, W.C. Characterization of model triacylglycerol (triolein, trilinolein and trilinolenin) autoxidation products via high-performance liquid chromatography coupled with atmospheric pressure chemical ionization mass spectrometry. J. Chromatogr. A 1998, 818, 169-186. [CrossRef]

19. Claxson, A.W.D.; Hawkes, G.E.; Richardson, D.P.; Naughton, D.P.; Haywood, R.M.; Chander, C.L.; Atherton, M.; Lynch, E.J.; Grootveld, M.C. Generation of lipid peroxidation products in culinary oils and fats during episodes of thermal stressing: A high field ${ }^{1} \mathrm{H}$ NMR study. FEBS Lett. 1994, 355, 81-90. [CrossRef]

20. Silwood, C.J.L.; Grootveld, M. Application of high-resolution two-dimensional ${ }^{1} \mathrm{H}$ and ${ }^{13} \mathrm{C}$ nuclear magnetic resonance techniques to the characterization of lipid oxidation products in autoxidized linoleoyl/linolenoyglycerols. Lipids 1999, 34, 741-756. [CrossRef]

21. Sacchi, R.; Falcigno, L.; Paduano, A.; Ambrosino, M.L.; Savarese, M.; De Giulio, B.; Addeo, F.; Paolillo, L. Quantitative evaluation of the aldehydes formed in heated vegetable oils using high resolution proton-NMR spectroscopy. Riv. Ital. Sostanze Grasse 2006, 83, 257-263.

22. Martınez-Yusta, A.; Goicoechea, E.; Guillen, M.D. A Review of thermo-oxidative degradation of food lipids studied by ${ }^{1} \mathrm{H}$ NMR spectroscopy: Influence of degradative conditions and food lipid nature. Compr. Rev. Food Sci. Food Saf. 2014, 13, 838-859. [CrossRef]

23. Grootveld, M.; Atherton, M.D.; Sheerin, A.N.; Hawkes, J.; Blake, D.R.; Richens, T.E.; Silwood, C.J.L.; Lynch, E.; Claxson, A.W.D. In vivo absorption, metabolism, and urinary excretion of $\alpha, \beta$-unsaturated aldehydes in experimental animals. Relevance to the development of cardiovascular diseases by the dietary ingestion of thermally-stressed polyunsaturate-rich culinary oils. J. Clin. Investig. 1998, 101, 1210-1218. [CrossRef] [PubMed]

24. Draper, H.H.; Polensek, L.; Hadley, M.; McGirr, L.G. Urinary malondialdehyde as an indicator of lipid peroxidation in the diet and in the tissues. Lipids 1984, 19, 836-843. [CrossRef]

25. Draper, H.H.; Csallany, A.S.; Hadley, M. Urinary aldehydes as indicators of lipid peroxidation in vivo. Free Radic. Biol. Med. 2000, 29, 1071-1077. [CrossRef]

26. Pignitter, M.; Somoza, V. Impact of dietary oxidized lipids on energy metabolism. Adv. Food Technol. Nutr. Sci. 2015, 1, 76-81. [CrossRef]

27. Penumetcha, M.; Khan, N.; Parthasarathy, S. Dietary oxidized fatty acids: An atherogenic risk? J. Lipid Res. 2000, 41, 1473-1480. [CrossRef]

28. Kritchevsky, D.; Tepper, S.A. Cholesterol vehicle in experimental atherosclerosis. 9. Comparison of heated corn oil and heated olive oil. J. Atheroscler. Res. 1967, 7, 647-651. [CrossRef]

29. Staprãns, I.; Rapp, J.H.; Pan, X.-M.; Hardman, D.A.; Feingold, K.R.; Oxidized lipids in the diet accelerate the development of fatty streaks in cholesterol-fed rabbits. Arterioscler. Thromb. Vasc. Biol. 1996, 16, 533-538. [CrossRef]

30. Marnett, L.J.; Hurd, H.K.; Hollstein, M.C.; Levin, D.E.; Esterbauer, H.; Ames, B.N. Naturally occurring carbonyl compounds are mutagens in Salmonella tester strain TA 104. Mutat. Res. 1985, 748, 25-34. [CrossRef]

31. Zhong, L.; Goldberg, M.S.; Parent, M.E.; Hanley, J.A. Risk of developing lung cancer in relation to exposure to fumes from Chinese-style cooking. Scand. J. Work Environ. Health 1999, 25, 309-316. [CrossRef] 
32. Shields, P.G.; Xu, G.X.; Blot, W.J.; Fraumeni, J.F., Jr.; Trivers, G.E.; Pellizzari, E.D.; Qu, Y.H.; Gao, Y.T.; Harris, C.C. Mutagens from heated Chinese and U.S. cooking oils. J. Natl. Cancer Inst. 1995, 87, 836-841. [CrossRef]

33. Wu, S.C.; Yen, G.C. Effects of cooking oil fumes on the genotoxicity and oxidative stress in human lung carcinoma (A-549) cells. Toxicol. In Vitro 2004, 18, 571-580. [CrossRef]

34. Young, S.C.; Chang, L.W.; Lee, H.L.; Tsai, L.H.; Liu, Y.C.; Lin, P. DNA damage induced by trans,trans-2,4-decadienal (tt-DD), a component of cooking oil fume, in human bronchial epithelial cells. Environ. Mol. Mutagenes. 2010, 51, 315-321.

35. Lee, T.; Gany, F. Cooking oil fumes and lung cancer: A review of the literature in the context of the U.S. population. J. Immigr. Minor. Health 2013, 15, 646-652. [CrossRef]

36. Indart, A.; Viana, M.; Grootveld, M.C.; Silwood, C.J.L.; Sanchez-Vera, I.; Bonet, B. Teratogenic actions of thermally-stressed culinary oils in rats. Free Radic. Res. 2002, 36, 1051-1058. [CrossRef] [PubMed]

37. Benedetti, A.; Ferrali, A.; Casini, A.F.; Peiri, S.; Comporti, M. Foot edema induced by carbonyl compounds originating from the peroxidation of microsomal lipids. Biochem. Pharmacol. 1980, 29, 121-124. [CrossRef]

38. Jayaraj, A.P.; Rees, K.R.; Tovey, F.E.I.; White, J.S. A molecular basis of peptic ulceration due to diet. Br. J. Exp. Pathol. 1986, 67, 149-155. [PubMed]

39. Long, E.K.; Murphy, T.C.; Leiphon, L.J.; Watt, J.; Morrow, J.D.; Milne, G.L.; Howard, J.R.H.; Picklo, M.J., Sr. Trans-4-hydroxy2-hexenal is a neurotoxic product of docosahexaenoic (22:6; n-3) acid oxidation. J. Neurochem. 2008, 105, 714-724. [CrossRef] [PubMed]

40. Leong, X.-F.; Mustafa, M.R.; Das, S.; Jaarin, K. Association of elevated blood pressure and impaired vasorelaxation in experimental Sprague-Dawley rats fed with heated vegetable oil. Lipids Health Dis. 2010, 9, 66. [CrossRef] [PubMed]

41. Emerit, I.; Khan, S.H.; Esterbauer, H. Hydroxynonenal, a component of clastogenic factors? Free Radic. Biol. Med. 1991, 10, 371-377. [CrossRef]

42. Emerit, I. Reactive oxygen species, chromosome mutation, and cancer: Possible role of clastogenic factors in carcinogenesis. Free Radic. Biol. Med. 1994, 36, 99-109. [CrossRef]

43. Carletto, C.; Nicolay, J.F.; Courbebaisse, Y. Oxidative stress and cutaneous ageing: The 'toxic second messengers' concept and an interesting family of products, 'pseudodipeptides'. Int. J. Cosmet. Sci. 2000, 22, 361-370. [CrossRef] [PubMed]

44. Opperman, M.; Benadé, A.; Abrecht, C.; Matsheka, L. South African seed oils are safe for consumption. S. Afr. J. Clin. Nutr. 2016, 29, 7-11. [CrossRef]

45. Karimi, S.; Wawire, M.; Mathooko, F.M. Impact of frying practices and frying conditions on the quality and safety of frying oils used by street vendors and restaurants in Nairobi, Kenya. J. Food Compos. Anal. 2017, 62, 239-244. [CrossRef]

46. Friere, P.C.M.; Lobo, L.C.B.; da Silva Freites, G.; de Castro Ferreria, T.A.P. The quality of deep-frying oils and fats used in street-fairs in Goiânia. Braz. Food Sci. Technol. 2013, 33, 569-576. [CrossRef]

47. Percival, B.C.; Savel, E.; Ampem, G.; Gibson, M.; Edgar, M.; Jafari, F.; Frederick, K.; Wilson, P.; Grootveld, M. Molecular composition of and potential health benefits offered by natural East African virgin sunflower oil products: A $400 \mathrm{MHz}{ }^{1} \mathrm{H} \mathrm{NMR}$ analysis study. Int. J. Nutr. 2019, 3, 22-43. [CrossRef]

48. Satyarthi, J.K.; Srinivas, D.; Ratnasamy, P. Estimation of free fatty acid content in oils, fats, and biodiesel by ${ }^{1} \mathrm{H}$ NMR spectroscopy. Energy Fuels 2009, 23, 2273-2277. [CrossRef]

49. Guillén, M.D.; Uriarte, P.S. Aldehydes contained in edible oils of a very different nature after prolonged heating at frying temperature: Presence of toxic oxygenated $\alpha, \beta$-unsaturated aldehydes. Food Chem. 2012, 131, 915-926. [CrossRef]

50. Grootveld, M.; Percival, B.C.; Moumtaz, S.; Grootveld, K.L. A ${ }^{1}$ H NMR-linked PCR modelling strategy for tracking the fatty acid sources of aldehydic lipid oxidation products in culinary oils exposed to simulated shallow-frying episodes. Int. J. Chem. Mol. Eng. 2019, 13, 251-263.

51. Parker, T.; Limer, E.; Watson, A.D.; Defernez, M.; Williamson, D.; Kemsley, E.K. $60 \mathrm{MHz}{ }^{1} \mathrm{H}-\mathrm{NMR}$ spectroscopy for the analysis of edible oils. Trends Anal. Chem. 2014, 57, 147-158. [CrossRef]

52. Siddiqui, N.; Sim, J.; Silwood, C.J.L.; Toms, H.; Iles, R.A.; Grootveld, M. Multicomponent analysis of encapsulated marine oil supplements using high resolution ${ }^{1} \mathrm{H}$ and ${ }^{13} \mathrm{C}$ NMR nuclear magnetic resonance (NMR) techniques. J. Lipid Res. 2003, 44, 2406-2427. [CrossRef]

53. Cortinas, L.; Galobart, J.; Barroeta, A.C.; Baucells, M.D.; Grashorn, M.A. Change in $\alpha$-tocopherol contents, lipid oxidation and fatty acid profile in eggs enriched with linolenic acid or very long-chain $w$-3 polyunsaturated fatty acids after different processing methods. J. Sci. Food Agric. 2008, 83, 820-829. [CrossRef]

54. Guillod, T. Interpolations, courbes de bézier et B-splines. Bull. Soc. Enseign. Neuchâtel. Sci. 2008, 34, 1-50.

55. Matthews, R.F.; Scanlan, R.A.; Libbey, L.M. Autoxidation products of 2,4-decadienal. J. Am. Oil Chem. Soc. 1971, 48, 745-747. [CrossRef]

56. Frankel, E.N. Frying fats. In Lipid Oxidation; Frankel, E.N., Ed.; The Oily Press Ltd.: Dundee, UK, 2005 ; pp. $227-248$.

57. Hrncirik, K.; Zeelenberg, M. Stability of essential fatty Acids and Formation of Nutritionally Undesirable Compounds in Baking and Shallow Frying. J. Am. Oil Chem. Soc. 2014, 91, 591-598. [CrossRef]

58. Mateos, R.; Uceda, M.; Aguilera, M.P.; Escuderos, M.E.; Maza, G.B. Relationship of Rancimat method values at varying temperatures for virgin olive oils. Eur. Food Res. Technol. 2006, 223, 246-252. [CrossRef]

59. Fanelli, S.; Zimmermann, T.E.G.; Salgado, H.R.N. FTIR Spectrophotometry as a green tool for quantitative analysis of drugs: Practical application to amoxicillin. J. Chem. 2018, 2018, 3920810. [CrossRef] 
60. Giese, E.; Rohn, S.; Fritsche, J. Chemometric tools for the authentication of cod liver oil based on nuclear magnetic resonance and infrared spectroscopy data. Anal. Bioanal. Chem. 2019, 411, 6931-6942. [CrossRef]

61. Xu, L.; Fei, T.; Yu, X.; Li, Q.; Liu, L. Qualitative analysis of edible oil oxidation by FTIR spectroscopy using mesh "cell". Anal. Methods 2015, 7, 4328-4333. [CrossRef]

62. Grootveld, M.; Percival, B.C.; Gibson, M.; Osman, Y.; Edgar, M.; Molinari, M.; Mather, M.L.; Casanova, F.; Wilson, P.B. Progress in low-field benchtop NMR spectroscopy in chemical and biochemical Analysis-A review. Anal. Chim. Acta 2019, 1067, 11-30. [CrossRef]

63. Saguy, I.S.; Shani, A.; Weinberg, P.; Garti, N. Utilization of jojoba oil for deep-fat frying of foods. Lebensm. Wiss. Technol. 1996, 29, 573-577. [CrossRef]

64. Sochr, J.; Cinkova, K.; Svorc, L. Degradation markers in nutritional products: A review. Aust. J. Anal. Pharm. Chem. 2014, 1, 1005.

65. Shahidi, F.; Wanasundara, U.N. Methods for measuring oxidative rancidity in fats and oils. In Food Lipids: Chemistry, Nutrition and Biotechnology; Akah, C.C., Min, D.B., Eds.; Marcel Dekker Inc.: New York, NY, USA, 2002; pp. $465-487$.

66. Chapter 19, Section 3: Spectroscopy of Aldehydes and Ketones. pp. 895-903. Available online: http://www.saplinglearning. com/media/loudon/loudon5ech19sec03.pdf (accessed on 2 March 2020).

67. Kalaliana, C.; Samira, B.; Rotha, E.; Chakira, A. UV absorption spectra of trans-2-pentenal, trans-2-hexenal and 2-methyl-2-pentenal. Chem. Phys. Lett. 2019, 718, 22-26. [CrossRef]

68. Cao, J.; Li, H.; Xia, X.; Zou, X.-G.; Li, J.; Zhu, X.-M.; Deng, Z.-Y. Effect of fatty acid and tocopherol on oxidative stability of vegetable oils with limited air. Int. J. Food Prop. 2015, 184, 808-820. [CrossRef]

69. Zhanyuan, D.; BramLmLage, W.J. Modified thiobarbituric acid assay for measuring lipid oxidation in sugar-rich plant tissue extracts. J. Agric. Food Chem. 1992, 40, 1566-1570. [CrossRef]

70. Hodges, D.; DeLong, J.; Forney, C.; Prange, R. Improving the thiobarbituric acid-reactive-substances assay for estimating lipid peroxidation in plant tissues containing anthocyanin and other interfering compounds. Planta 1999, 207, 604-611. [CrossRef]

71. Hazard Symbols and Hazard Pictograms-Chemical Classification-HSE. Available online: http://www.hse.gov.uk (accessed on 11 March 2020).

72. Mehta, U.; Swinburn, B. A review of factors affecting fat absorption in hot chips. Crit. Rev. Food Sci. Nutr. 2001, 41, 133-154. [CrossRef] [PubMed]

73. Moreira, R.G.; Castell-Perez, M.E.; Barrufet, M.A. Deep-Fat Frying Fundamentals and Applications; Aspen Publishers Inc.: Gaithersburg, MD, USA, 1999.

74. Mellema, M. Mechanism and reduction of fat uptake in deep-fat fried foods. Trends Food Sci. Technol. 2000, 14, 364-373. [CrossRef]

75. Alireza, S.; Tan, C.P.; Hamed, M.; Che Man, Y.B. Effect of frying process on fatty acid composition and iodine value of selected vegetable oils and their blends. Int. Food Res. J. 2010, 17, 295-302.

76. Naseri, M.; Abedi, E.; Mohammadzadeh, B.; Afsharnaderi, A. Effect of frying in different culinary fats on the fatty acid composition of silver carp. Food Sci. Nutr. 2013, 1, 292-297. [CrossRef]

77. Csallany, A.S.; Han, I.; Shoeman, D.W.; Chen, C.; Yuan, J. 4-Hydroxynonenal (HNE), a toxic aldehyde in french fries from fast food restaurants. JAOCS 2015, 92, 1413-1419. [CrossRef]

78. Powers, S.J.; Mottram, D.S.; Curtis, A.; Halford, N.G. Acrylamide levels in potato crisps in Europe from 2002 to 2016. Food Add. Contam. Part A 2017, 34, 2085-2100. [CrossRef]

79. Krishnakumar, T.; Visvanathan, R. Acrylamide in food products: A review. J. Food Process. Technol. $2014,5,7$.

80. Ehling, S.; Hengel, M.; Shibamoto, T. Formation of acrylamide from lipids. Adv. Exp. Med. Biol. 2005, 56, $223-233$.

81. Acceptable Daily Intakes for Agricultural and Veterinary Chemicals: Current as of 31 March 2016; The Office of Chemical Safety, Department of Health: Sydney, Australia, 2016.

82. World Health Organization; Gomes, R.; Meek, M.E. Concise International Chemical Assessment Document 43 ACROLEIN; United Nations Environment Programme, the International Labour Organization, and the World Health Organization: Geneva, Switzerland, 2002.

83. Grootveld, M.; Percival, B.C.; Grootveld, K.L. Chronic non-communicable disease risks presented by lipid oxidation products in fried foods. Hepatobil. Surg. Nutr. 2018, 7, 305-312. [CrossRef]

84. van Andel, I.; Sleijffers, A.; Schenk, E.; Rambali, B.; Wolterink, G.; van de Werken, G.; van Aerts, L.A.G.J.M.; Vleeming, W.; van Amsterdam, J.G.C. Adverse Health Effects of Cigarette Smoke: Aldehydes Crotonaldehyde, Butyraldehyde, Hexanal, and Malonaldehyde; RIVM Report 340630002/2006; RIVM: Bilthoven, The Netherlands, 2006.

85. National Institute of Occupational Safety and Health's (NIOSH's). Immediately Dangerous to Life or Health (IDLH) Concentrations; CAS Number 107-02-08; Centers for Disease Control and Prevention, NIOSH Publications and Products: Atlanta, GA, USA, 1994.

86. Henderson, Y.; Haggard, H.W. Noxious Gases, 2nd ed.; Reinhold Publishing Corporation: New York, NY, USA, 1943 ; p. 138.

87. NRC. Emergency and Continuous Exposure Limits for Selected Airborne Contaminants; National Academy Press, Committee on Toxicology, Board on Toxicology and Environmental Health Hazards, Commission on Life Sciences, National Research Council: Washington, DC, USA, 1984; Volume 1, pp. 27-34.

88. Darley, E.F.; Middleton, J.T.; Garber, M.J. Plant damage and eye irritation from ozone-hydrocarbon reactions. J. Agric. Food Chem. 1960, 8, 483-485. [CrossRef]

89. Sim, V.M.; Pattle, R.E. Effect of possible smog irritants on human subjects. J. Am. Med. Assoc. 1957, 165, 1908-1913. [CrossRef] [PubMed] 
90. Japan Bioassay Research Center. Summary of Inhalation Carcinogenicity Study of Acrolein in B6D2F1 Mice; Japan Organization of Health and Safety: Kanagawa, Japan, 2016.

91. Japan Bioassay Research Center. Summary of Inhalation Carcinogenicity Study of Acrolein in F344 Rats; Japan Organization of Health and Safety: Kanagawa, Japan, 2016.

92. Nath, R.G.; Ocando, J.E.; Guttenplan, J.B.; Chung, F.L. 1,N2-propanodeoxyguanosine adducts: Potential new biomarkers of smoking-induced DNA damage in human oral tissue. Cancer Res. 1998, 58, 581-584. [PubMed]

93. Chen, H.J.; Lin, W.P. Quantitative analysis of multiple exocyclic DNA adducts in human salivary DNA by stable isotope dilution nanoflow liquid chromatography-nanospray ionization tandem mass spectrometry. Anal. Chem. 2011, 83, 8543-8551. [CrossRef] [PubMed]

94. Feng, Z.; Hu, W.; Hu, Y.; Tang, M.S. Acrolein is a major cigarette-related lung cancer agent: Preferential binding at p53 mutational hotspots and inhibition of DNA repair. Proc. Natl. Acad. Sci. USA 2006, 103, 15404-15409. [CrossRef] [PubMed]

95. Chung, F.L.; Tanaka, T.; Hecht, S.S. Induction of liver tumors in F344 rats by crotonaldehyde. Cancer Res. 1986, 46, 1285-1289. [PubMed]

96. Dittberner, U.; Eisenbrand, G.; Zankl, H. Genotoxic effects of the alpha,beta-unsaturated aldehydes 2-trans-butenal, 2-transhexenal and 2-trans, 6-cis-nonadienal. Mutat. Res. 1995, 335, 259-265. [CrossRef]

97. IARC Monograph Working Group. Carcinogenicity of acrolein, crotonaldehyde, and arecoline. Lancet Oncol. 2020. [CrossRef]

98. Pamies, D.; Vilanova, E.A.; Wexler, P. Encyclopedia of Toxicology, 3rd ed.; Academic Press: Cambridge, MA, USA, 2014; pp. 63-68; ISBN 9780123864550. [CrossRef]

99. Esterbauer, H.; Ertl, A.; Scholz, N. The reaction of cysteine with $\alpha, \beta$-unsaturated aldehydes. Tetrahedron 1976, 32, 285-289. [CrossRef]

100. Allam, S.S.M.; Mohamed, H.M.A. Thermal stability of some commercial natural and synthetic antioxidants and their mixtures. J. Food Lipids 2002, 9, 277-293. [CrossRef]

101. Battino, M.; Quiles, J.L.; Huertas, J.R.; Ramirez-Tortosa, M.C.; Cassinello, M.; Mañas, M.; Lopez-Frias, M.; Mataix, J. Feeding fried oil changes antioxidant and fatty acid pattern of rat and affects rat liver mitochondrial respiratory chain components. J. Bioenerget. Biomem. 2002, 34, 127-134. [CrossRef]

102. Quiles, J.L.; Huertas, J.R.; Battino, M.; Ramirez-Tortosa, M.C.; Cassinello, M.; Mataix, J.; Lopez-Frias, M.; Mañas, M. The intake of fried virgin olive or sunflower oils differentially induces oxidative stress in rat liver microsomes. Br. J. Nutr. 2002, 88, 57-65. [CrossRef] [PubMed]

103. Andrikopoulos, N.K.; Dedoussis, G.V.Z.; Falirea, A.; Kalogeropoulos, N.; Hatzinikola, H.S. Deterioration of natural antioxidant species of vegetable edible oils during the domestic deep-frying and pan-frying of potatoes. Int. J. Food Sci. Nutr. 2002, 53, 351-363. [CrossRef]

104. Percival, B.C.; Zbasnik, R.; Schlegel, V.; Edgar, M.; Zhang, J.; Grootveld, M. Determinations of the peroxidative susceptibilities of cod liver oils by a newly-developed ${ }^{1} \mathrm{H}$ NMR-based method: Resistance of an antioxidant-fortified product isolated from pre-fermented sources. Biomed. Cent. Res. Notes 2020, 13, 73. [CrossRef]

105. Mozaffarian, D.; Aro, A.; Willett, W.C. Health effects of trans-fatty acids: Experimental and observational evidence. Eur. J. Clin. Nutr. 2009, 63, S5-S21. [CrossRef]

106. Song, J.; Park, J.; Jung, J.; Lee, C.; Gim, S.Y.; Ka, H.; Yi, B.R.; Kim, M.-J.; Kim, C.-I.; Lee, J.H. Analysis of trans fat in edible oils with cooking process. Toxicol. Res. 2015, 31, 307-312. [CrossRef]

107. Countdown to 2023: WHO Report on Global Trans-Fat Elimination 2020; World Health Organization: Geneva, Switzerland, 2020; Licence: CC BY-NC-SA 3.0 IGO. Available online: https:/ /apps.who.int/iris/bitstream/handle/10665/334170/9789240010178 -eng.pdf (accessed on 9 December 2020). 